\title{
Blood-brain barrier dysfunction in aging induces hyper-activation of TGF-beta signaling and chronic yet reversible neural dysfunction
}

Senatorov, V.V. Jr. ${ }^{1,2 *}$, Friedman, A.R. ${ }^{1,2 *}$, Milikovsky, D.Z. ${ }^{3}$, Ofer, J. ${ }^{3}$, Saar-Ashkenazy, R. ${ }^{3}$, Charbash, A. ${ }^{3}$, Jahan, N. ${ }^{2,4}$, Chin, G. ${ }^{4}$, Mihaly, E. ${ }^{5}$, Lin, J.M. ${ }^{2}$, Ramsay, H.J. ${ }^{2}$, Moghbel, A. ${ }^{4}$, Preininger, M.K. ${ }^{4}$, Eddings, C.R. ${ }^{4}$, Harrison, H.V. ${ }^{6}$, Patel, R. ${ }^{4}$, Shen, $Y .{ }^{4}$, Ghanim, H. ${ }^{4}$, Sheng, H. ${ }^{2}$, Veksler, R. ${ }^{3}$, Sudmant, P.H. ${ }^{2}$, Becker, A. ${ }^{7}$, Hart, B. ${ }^{8}$, Rogawski, M.A. ${ }^{9}$, Dillin, A. ${ }^{10}$, Friedman, A. ${ }^{3,11,+}$, and Kaufer, D. ${ }^{1,2,12,+, \neq}$

${ }^{1}$ Helen Wills Neuroscience Institute and Berkeley Stem Cell Center, University of California, Berkeley, Berkeley, CA 94720, USA

${ }^{2}$ Department of Integrative Biology, University of California, Berkeley, Berkeley, CA 94720, USA

${ }^{3}$ Departments of Physiology and Cell Biology, Cognitive and Brain Sciences, Zlotowski Center for Neuroscience, Ben-Gurion University of the Negev, Beer-Sheva 84105, Israel

${ }^{4}$ Department of Molecular and Cell Biology, University of California, Berkeley, Berkeley, CA 94720, USA

${ }^{5}$ Department of Bioengineering, University of California, Berkeley, Berkeley, CA 94720, USA

${ }^{6}$ School of Public Health, University of California, Berkeley, Berkeley, CA 94720, USA

${ }^{7}$ Section for Translational Epilepsy Research, Department of Neuropathology, University of Bonn Medical Center, Bonn, Germany

${ }^{8}$ Innovation Pathways, Palo Alto, CA 94301

${ }^{9}$ Department of Neurology, School of Medicine, University of California, Davis, Sacramento, CA 95817, USA

${ }^{10}$ The Glenn Center for Aging Research, Howard Hughes Medical Institute, Department of Molecular and Cell Biology, University of California, Berkeley, Berkeley, CA 94720, USA.

${ }^{11}$ Department of Medical Neuroscience and Brain Repair Center, Dalhousie University, Halifax, NS, Canada B3H4R2

${ }^{12}$ Canadian Institute for Advanced Research, Toronto, ON, Canada M5G1M1

* These authors contributed equally to this work

${ }^{+}$Senior authors contributed equally to this work

$\ddagger$ Corresponding author

\section{Abstract}

Aging involves a decline in neural function that contributes to cognitive impairment and disease. However, the mechanisms underlying the transition from a young-and-healthy to aged-anddysfunctional brain are not well understood. Here, we report breakdown of the vascular blood-brain barrier (BBB) in aging humans and rodents, which begins as early as middle age and progresses to the end of the lifespan. Gain-of-function and loss-of-function manipulations show that this BBB dysfunction triggers hyperactivation of transforming growth factor $\beta$ (TGF $\beta$ ) signaling in astrocytes, which is necessary and sufficient to cause neural dysfunction and age-related pathology. Specifically, infusion of the serum protein albumin into the young brain (mimicking BBB leakiness) induced astrocytic TGF $\beta$ signaling and an aged brain phenotype including aberrant electrocorticographic activity, vulnerability to seizures, and cognitive impairment. Furthermore, conditional genetic knockdown of astrocytic TGF $\beta$ receptors, or pharmacological inhibition of TGF $\beta$ signaling, reversed these symptomatic outcomes in aged mice. Finally, we found that this same signaling pathway is activated in aging human subjects with BBB dysfunction. Our study identifies dysfunction in the neurovascular unit as one of the earliest triggers of neurological aging, and demonstrates that the aging brain may retain considerable latent capacity which can be revitalized by therapeutic inhibition of TGF $\beta$ signaling. 


\section{Introduction}

Aging involves cognitive deterioration which poses an increasing healthcare burden on today's society with its prolonged life expectancy. Despite calls for a better understanding of brain aging and new therapeutic targets, the underlying mechanisms that cause decline in neural function in aging are still not understood. We approached this topic by exploring the causal involvement of the blood-brain barrier (BBB) pathology in age-related brain dysfunction. The BBB is a tightly-regulated interface composed of specialized endothelial cells, pericytes and astrocytic end-feet that form a protective sheath around brain capillaries. By restricting the free diffusion of blood-borne molecules, the BBB establishes a sequestered brain microenvironment, including the precisely balanced ionic concentrations needed for neural activity, the compartmentalization of brain-specific growth factors and signaling molecules, and the immune-privileged brain environment $(1,2)$. Thus, the BBB is a fundamental and essential component of healthy brain function.

Alarming observations of widespread BBB breakdown in aging patients were first reported in the 1970s (3), raising the possibility that vascular leakiness and infiltration of toxic blood-borne molecules into the brain could cause neural impairments and contribute to diseases such as dementia $(2,4-7)$. This theory has been primarily supported by human clinical evidence showing that BBB breakdown in aging individuals is strongly correlated with cognitive decline and Alzheimer's disease (7-12). Furthermore, this association has been localized to functional brain sub-regions: BBB breakdown specifically in the hippocampus is associated with significant mild cognitive impairment (13) - suggesting that BBB dysfunction could be a cause of impairment within impacted tissues. However, these human studies have also yielded contradictory results, are complicated by variability in population and study methodology $(14,15)$, and are inherently correlative. There have been very few rodent studies that assess BBB status in aging (16), and to our knowledge, no studies that test mechanistic hypotheses about how BBB breakdown affects brain function in aging. A mechanistic understanding of the biological consequences of BBB breakdown is critical to discern whether the association between BBB dysfunction and cognitive decline is spurious, correlative, or causal. Furthermore, mechanistic insights would have the potential to reveal new druggable targets for the intractable health problems of age-related cognitive decline and dementia. Indeed, an improved understanding of vascular contributions to cognitive decline and dementia has been designated as one of the highest priority research areas for new breakthroughs in Alzheimer's disease and related dementias (17).

While little is known about the consequences of BBB breakdown in aging, there are several other disease contexts that involve BBB dysfunction, which could reveal relevant candidate mechanisms. In particular, traumatic brain injury (TBI) not only causes severe BBB breakdown (18-20), but also involves secondary symptoms of cognitive impairment and increases risk for dementia. In rodent models of TBI and leaky BBB, blood-borne proteins that infiltrate into the brain cause a robust injury response by activating the transforming growth factor beta (TGF $\beta$ ) signaling pathway. Key mediators of this response include the serum protein fibrinogen, which carries and releases latent TGF $\beta$ in the brain (21), and albumin, which binds to the TGF $\beta$ receptor and activates signaling (22). In both cases, astrocytes act as the primary responders that detect the blood-borne ligands and transduce TGF $\beta$ signaling (21-23). In turn, activated astrocytes release inflammatory cytokines and more $\operatorname{TGF} \beta 1(22,24,25)$, form glial scars (21), and remodel neural circuits to cause hyperexcitability and dysfunction (22, 24, 26-28). These studies point to the TGF $\beta$ signaling pathway as a candidate mechanism that could play a role in pathological outcomes following BBB breakdown in aging. To test this hypothesis, we used genetic and 
pharmaceutical interventions to test the causality of BBB dysfunction and TGF $\beta$ signaling in progressive neural dysfunction across the lifespan of naturally aging mice.

\section{Results}

\section{Progressive BBB dysfunction and albumin extravasation in the hippocampus starts in middle age}

To establish the time course of age-related BBB decline, we quantified extravasation of serum albumin, an established marker of BBB permeability (29-31) which also plays a mechanistic role in triggering TGF $\beta$ signaling (22). We focused our analysis on the hippocampus - a key brain region associated with agerelated memory decline (13). Albumin was effectively absent from the hippocampus of young mice, but was first detectable in the aging hippocampus starting as early as 12 months ("middle age") and consistently elevated in aging up to two years, near the end of the lifespan (Fig. 1A and Fig. S1A). These results indicate that $\mathrm{BBB}$ dysfunction appears earlier than has been appreciated, placing it among the earliest known biomarkers of aging in the rodent brain. The onset of BBB dysfunction at around 12 months corresponds with other early biological hallmarks of aging, including the typical onset of reproductive senescence in female mice, as well as the earliest appearance of mild cognitive impairments in various behavior tasks (32-37). To confirm age-related BBB dysfunction, we raised a separate cohort of aged mice and used an alternate method to quantify BBB permeability, based on detecting leakage of a fluorescent tracer, Evans blue (EB), into the brain after i.v. injection (38). Compared to 2-4 month old young mice, 12 and 21-24 month old aged mice showed significantly elevated levels of EB leakage into the brain (Fig. S1B-C), thus reproducing our finding of age-related BBB breakdown by an independent method.

\section{Age-dependent albumin accumulation is cell-type specific}

Albumin is known to accumulate in astrocytes when it extravasates through a dysfunctional BBB $(22,24$, 39). We therefore used a transgenic mouse line that comprehensively labels astrocytes via the Aldh1L1 (Aldehyde Dehydrogenase 1 Family Member L1) promoter (Rep-Aldh1L1) (40, 41). Expression of this marker was stable and did not change across the lifespan (Fig. S1D). To estimate the specificity of albumin uptake in different cell types, we performed immunostaining for markers of microglia (Iba1), oligodendrocytes (CAII), and neurons (NeuN), and quantified the percent of all albumin+ cells for each cell type in the aged (18-24 months) mouse hippocampus. Albumin co-localized predominantly with Aldh1L1-positive astrocytes, accounting for approximately $60 \%$ of albumin-labeled cells, while each of the other cell types co-labeled approximately $20 \%$ of all albumin-positive cells (Fig. S1E). Concurrent with the time course of overall albumin extravasation and BBB dysfunction, significant astrocytic uptake of albumin was first detected at 12 months, and further increased with age up to 21 months old (Fig. 1C).

\section{Age-dependent activation of aberrant TGF $\beta$ signaling in astrocytes}

Albumin endocytosis into astrocytes is mediated by binding to the TGF $\beta$ receptor II (TGF $\beta$ R) subunit, which in turn activates the TGF $\beta$ RI ALK5 $(22,28)$ to induce phosphorylation of Smad2 (pSmad2) and carry out signal transduction of the ALK5-TGF $\beta$ signaling cascade. Further, this activation also increases the production of TGF $\beta 1$ in astrocytes (Weissberg et al., 2015) and activation of latent TGF $\beta 1$ protein from extra-cellular matrix (26), yielding an increase in the canonical ligand of TGF $\beta R$ and therefore amplification of the TGF $\beta$ cascade. Hence, we next investigated the relationship between albumin uptake and TGF $\beta$ signaling in the aged mouse brain by quantifying immunolabeled phosphorylated 
Smad2 protein (pSmad2). Concurrent with the time course of albumin extravasation, aging mice showed a progressive increase in the levels of pSmad2 co-localized with albumin-positive astrocytes (Fig. 1B and D). Activation of the TGF $\beta$ pathway was further quantified by Western blot, showing increased levels of pSmad 2 in the hippocampus of old mice, compared to young, and increased levels of active TGF $\beta 1$, a positive feedback output of the TGF $\beta$ pathway (Fig. 1E).

\section{Network hyperexcitability in aged mice}

Based on the finding that BBB dysfunction and TGF $\beta$ signaling causes hyperexcitability after head injury $(22,24,26-28,39)$, we hypothesized that similar hyperexcitability may be triggered by BBB decline and contribute to cognitive impairment in aging mice. Indeed, hippocampal hyperexcitability is an early biomarker of mild cognitive impairment in humans that precedes progression to $A D(42,43)$, and is also an early marker of disease progression in rodent $A D$ models $(44,45)$. Thus, we used the pentylenetetrazol (PTZ) seizure assay to investigate the time course for onset and progression of hyperexcitability in aging mice. In this assay, injection of PTZ, a non-competitive GABA receptor blocker, induces seizures that have been shown to be a reliable readout of underlying hippocampal hyperexcitability associated with aging and $A D$ models (45) (i.e. mice with higher hyperexcitability exhibit lower induced seizure threshold). Compared to 3 month old mice, aged mice showed increased severity of induced seizures (quantified based on rate of progression through the Racine seizure severity scale, Fig. S1F), beginning at the 12-month-old time point (Fig. 1F). Furthermore, old mice were highly vulnerable to mortality from severe seizures, with a significantly shorter latency to mortality (Fig. 1G).

\section{Aberrant paroxysmal slow wave events in aged mice}

Next, we sought to measure and characterize hyperexcitability directly via electrophysiology. To do so, we recorded telemetric electrocorticography (ECoG) using epidural electrodes implanted in young ( 3 months old) and old (18-24 months old) mice over a period of 5 days in the home cage. We found that aged mice showed an increase in the relative power of slow wave activity $(<5 \mathrm{~Hz}$ ) (Fig. S1G), similar to EEG slowing described in human dementia patients (46-48), which is thought to reflect dysfunctional neural networks. Detailed analysis of this aberrant ECoG signal revealed that the slow-wave activity was not continuous, but rather manifested in discrete, transient paroxysmal slow wave episodes (pSWEs, median frequency $<5 \mathrm{~Hz}$; Fig. $1 \mathrm{H}$ ), which were significantly elevated in aged mice relative to young (Fig. 11). These pSWEs, characterized by a median power of less than $5 \mathrm{~Hz}$ over 10 consecutive seconds, were similar to paroxysmal ECoG events that have been observed in epileptogenic animals that have hippocampal hyperexcitability (49). Given the potential association between hyperexcitability and seizures, we also used an automated seizure detection algorithm $(24,49)$ to search for spontaneous seizures in the ECoG recordings, and found that aged mice had no seizure events and were not epileptic. Thus, the pSWEs constitute distinct subclinical paroxysmal events that are associated with hyperexcitability.

Together, our initial studies showed that BBB breakdown and astrocytic TGF $\beta$ signaling occur at early stages of mouse brain aging, concurrent with hyperexcitability and neural network dysfunction. Next, we performed comparative human studies to investigate whether similar BBB dysfunction and TGF $\beta$ signaling is also present in aging human subjects.

The aging human brain shows progressive BBB dysfunction associated with astrocytic TGF $\beta$ signaling 
In our rodent studies, we directly measured the progression of albumin extravasation and TGF $\beta$ signaling across the lifespan. BBB dysfunction has also been reported in aging humans including mild cognitive impairment ( $\mathrm{MCl}$ ) and Alzheimer's disease and related dementia (ADRD) patients, using indirect measures such as CSF sampling or imaging, which can be technically challenging and may produce conflicting results (15). Thus, we sought to further quantify age-related BBB dysfunction in human subjects, expanding on results reported elsewhere $(8,10,12,13)$. We used dynamic contrastenhanced MRI scanning (DCE-MRI) to quantify BBB permeability in 113 healthy human subjects with an age range of 21 to 83 years old (Fig. 2A; Table S1). We first established normal levels of brain permeability based on permeability values in healthy, young patients (age 21-40), setting an upper value for "normal permeability" at the $95^{\text {th }}$ percentile (i.e. $95 \%$ of brain voxels in the averaged healthy young brain were below this value). Based on this threshold, we constructed permeability maps for each subject and quantified the percentage of "leaky" voxels in the whole brain (Fig. 2A). This analysis revealed a significant linear increase in BBB permeability in aging (Fig. S2A). To estimate the overall prevalence of BBB dysfunction, we further categorically classified individuals as either BBB-intact (BBB-I) or BBB-disrupted (BBB-D; permeability in more than $5 \%$ of brain volume). By age 60 , nearly half of the population was affected by BBB-D (Fig. S2B).

BBB breakdown, specifically in the hippocampus, has been associated with the incidence of mild cognitive impairment (13). Furthermore, hippocampal atrophy and in particular asymmetrical atrophy are early biomarkers that predict the transition from cognitively normal to $\mathrm{MCI}$ to ADRD (50-55). Thus, we used anatomical localization to specifically assess BBB permeability in the left and right hippocampus, and found that aging individuals showed asymmetric BBB breakdown localized to the right hippocampus (Fig. 2B). Given that our study was comprised of cognitively "normal" aging individuals, excluding subjects that met the diagnostic criteria of mild cognitive impairment, this suggests that BBB breakdown may be a very early aging event that precedes cognitive decline and neuropathology.

We next sought to complement the indirect MRI approach by directly assessing BBB dysfunction, and its association with astrocytic TGF $\beta$ signaling in aging human brains. We examined post-mortem tissue from young ( $31.3 \pm 5$ years old) and aging ( $70.6 \pm 5.6$ years) human subjects with no history of brain disorder (Table S2), and directly measured co-localization of albumin extravasation and TGF $\beta$ signaling, in astrocytes as performed in our rodent studies. We found high levels of the serum protein albumin in the old hippocampus, which was absent in the young (Fig. 2C). Albumin was detected in astrocytes (identified by the astrocytic marker GFAP), and colocalized with phosphorylated Smad2 (pSmad2), the primary signaling protein of the canonical TGF $\beta$ signaling cascade. The number of albumin-pSmad 2 colabeled astrocytes was significantly increased in old vs. young individuals (Fig. 2D).

This immunostaining indicated that BBB dysfunction and albumin induces TGF $\beta$ signaling in the aging human hippocampus, as observed in our aging rodent studies. In turn, this would be predicted to cause positive feedback and elevated levels of TGF $\beta$. To assess this, we obtained human brain transcriptome data from the publicly available Genotype-Tissue Expression (GTEx) project (56), with data from 123 human subjects ranging in age from 20 to 70 years old, and investigated brain expression levels of the major TGF $\beta$ isoforms (TGF $\beta 1,2$, and 3 ) in the human hippocampus. These data showed that hippocampal expression of TGF $\beta 1$ and TGF 33 significantly increase with age in human subjects (Fig. 2E).

These studies showed that BBB dysfunction and TGF $\beta$ signaling are early biomarkes of aging in both rodents and humans, and in rodents are associated with symptomatic hyperexcitability. Next, we used 
gain-of-function and loss-of-function interventions to determine whether albumin-induced TGF $\beta$ signaling plays a causal role in age-related symptoms of neural dysfunction and cognitive impairment

\section{Infusion of albumin into young brains causes hyperexcitability and paroxysmal slow wave events}

To test if TGF $\beta$ signaling, as induced by BBB dysfunction, is sufficient to cause symptomatic pathology associated with aging, we infused albumin (iAlb) or control artificial cerebrospinal fluid (aCSF) into the brain ventricles of healthy, young adult rats and mice via osmotic mini-pump (Fig. S3A). Following infusion, the exogenous albumin diffused readily into the ipsilateral hippocampus, and was taken up by astrocytes within 48 hours of infusion (Fig. S3A-B). We then assessed outcomes in young rodents (Fig. $3 \mathrm{~A})$, to determine if iAlb is sufficient to cause aged-like symptoms of network and cognitive dysfunction. We conducted the hyperexcitability PTZ seizure assay in young mice 48 hours after iAlb. The iAlb mice showed significantly increased seizure severity and mortality induced by PTZ compared to aCSF controls (Fig. 3B-C; Fig. S3C), fully reprising the hyperexcitable seizure vulnerability observed in naturally aged mice. Furthermore, we directly investigated network dysfunction via recordings of ECoG activity in young iAlb rats, and found that iAlb caused a symptomatic slowing of ECoG activity and a significantly increased pSWEs (Fig. S3D-G; Fig. 3D) similar to the aberrant activity observed in aged rodents. This elevated level of pSWEs was observed only in recordings from the ipsilateral hemisphere receiving iAlb infusion, but not in the contralateral hemisphere, indicating specificity of the aberrant neural activity to the tissue affected by iAlb.

\section{Infusion of albumin into young brains causes impaired memory performance}

We next investigated whether iAlb is sufficient to cause cognitive impairment in young mice. We implanted mice with iAlb (or aCSF control) osmotic pumps for one week. One month later, we tested mice in the Morris water maze (MWM) spatial memory task. Mice that received iAlb infusion were significantly impaired in memory performance over 9 days of MWM training, compared to control mice with aCSF implant (Fig. 3E). Together, these data show that iAlb is sufficient to induce TGF $\beta$ signaling and confer a dramatic "old age" phenotype in young rodents, including aberrant neural ECoG activity, hyperexcitable seizure vulnerability, and cognitive impairment.

\section{Genetic knockdown of astrocytic TGF $\beta$ signaling reverses pathological outcomes in aging mice}

To test the causal role of astrocytic TGF $\beta$ signaling in age-related impairments, we generated a transgenic mouse line (aTGF $\beta R 2 / K D$ ) expressing inducible Cre recombinase under the astrocyte-specific GLAST promoter. This enables conditional knockout of the floxed (fl) TGF $\beta R$ with temporal precision, specifically in astrocytes (Fig. 4A), allowing us to interrogate the role of astrocytic TGF $\beta$ signaling in mediating pathological outcomes. Treatment with tamoxifen (tam) induced efficient recombination in approximately $40 \%$ of hippocampal astrocytes but not in neurons (Fig. S4A-C), and significantly reduced levels of TGF $\beta R$ (Fig. S4D), thus effectively causing knockdown (KD) to inhibit but do not fully abolish TGF $\beta$ signaling. We aged cohorts of aTGF $\beta R 2 / K D$ mice to early (12-16 months) and late (17-24 months) stages of aging, and then induced TGF $\beta R 2$ KD in astrocytes to test their role in age-related hyperexcitability and cognitive dysfunction (Fig. 4B). Following induction, aged KD mice showed a significant decrease in hippocampal pSmad2 protein and reduction in expression of TGF $\beta$ R2 (Fig. S4E-F).

In early aging (12-16 months), mice with homozygous induced KD of the aTGF $\beta R 2$ ( $\mathrm{fl} / \mathrm{fl})$ were protected against symptomatic hyperexcitability, showing a low level of vulnerability to PTZ-induces seizures and seizure mortality (Fig. 4C; Fig. S4G) that was similar to young mice. In contrast, 12-16 month old control 
mice that were heterozygous for floxed TGF $\beta R 2(\mathrm{fl} /+)$ (and hence retained intact astrocytic TGF $\beta$ signaling), or that were homozygous for floxed TGF $\beta R 2$ but injected with vehicle instead of tamoxifen (hence no KD induction; aTGF $\beta R 2+/+$ ) showed significantly elevated levels of seizure vulnerability and mortality (Fig. 4C). The KD intervention was also effective at late aging timepoints: 17-21 month aged aTGF $\beta 22$ heterozygote $(\mathrm{fl} /+)$ controls showed typical age-related vulnerability to induced seizures, whereas aTGFBR2 KD (fl/fl) displayed low vulnerability and mortality to PTZ challenge (Fig. 4D; Fig. S4H). These results show that genetic KD of astrocytic TGF $\beta$ signaling is sufficient to reverse symptoms of hyperexcitability in the PTZ assay, at both early and late stages of aging.

\section{Genetic knockdown of astrocytic TGF $\beta$ signaling reverses cognitive impairments in aging mice}

To assess the role of astrocytic TGF $\beta$ signaling in age-related cognitive decline, we tested aged transgenic mice for spontaneous alternation in the T-maze, a hippocampal working memory task (57) that is impaired in aging rodents (58). The task is optimal for assessing aging rodents because it can be performed rapidly without extensive training, is sensitive to mild impairments in hippocampal function $(59,60)$, and yet it is relatively unaffected by motor and vision impairments that may confound aging mice in traditional tasks such as Morris Water maze (57). At both early and late aging time points, aTGF $\beta R 2$ KD mice made significantly more correct choices in the T-maze task, indicating improved working memory compared to heterozygous controls (Fig. 4E-F).

While aTGF $\beta$ R2 KD was effective at improving cognitive function in both early and late stages, we also found greater heterogeneity in cognitive scores in the early (12-16 month) aging group - as would be expected for an early stage of aging in which some mice may have transitioned into mild cognitive impairment while other remain cognitively healthy. To investigate this individual variability, we performed T-maze in an additional cohort of early aging (12-16 month old) aTGF 322 KD and heterozygous controls, and collected dissected hippocampi to quantify the relationship individual cognitive scores and pSmad2, the molecular marker of TGF $\beta$ signaling. Across both heterozygous $(\mathrm{fl} /+)$ and homozygous $(\mathrm{fl} / \mathrm{fl}$ ) genotypes, pSmad2 levels were negatively correlated with T-maze performance (Fig. 4G), providing further evidence for the role of TGF $\beta$ signaling in cognitive impairment.

Together, these results show that targeted inhibition of the TGF $\beta$ signaling pathway, via induced KD in astrocytes, is sufficient to reverse the outcomes of seizure vulnerability and cognitive impairment in a hippocampal spatial working memory task in old mice, and that cognitive outcomes in a heterogeneous "mildly impaired" early aging cohort are correlated with the individual levels of TGF $\beta$ signaling.

\section{A novel small molecule TGFßR1 kinase inhibitor blocks iAlb-induced effects in the young brain}

Our findings in mice and human brains support an evolutionarily conserved role of astrocytic TGF $\beta$ signaling in the pathogenesis of age-related neurological vulnerability, further indicating a therapeutic potential in targeting TGF $\beta$ R. Thus, we next tested the efficacy of a novel small molecule TGF $\beta R 1$ kinase inhibitor, IPW (61). IPW has a promising clinical profile - including the ability to cross the BBB and good stability following oral dosing (Fig. S5A), making it suitable for once-per-day dosing to achieve inhibition of TGF $\beta R$ signaling (Rabender et al., 2016). We first tested IPW in young mice with TGF $\beta$ signaling induced by iAlb, treating them with daily i.p. injections $(20 \mathrm{mg} / \mathrm{kg}$ ) of IPW for two days after pump implant. IPW treatment significantly reduced pSmad2 levels measured in the dissected hippocampus of treated mice, compared to mice treated with vehicle control (Fig. 5A-B). When examining the vulnerability to PTZ, iAlb mice treated with vehicle showed high seizure vulnerability, replicating our previous results, whereas IPW treatment effectively reversed this vulnerability, reducing both seizure 
severity and mortality to the level seen in the control aCSF infused mice (Fig. 5C-D; Fig. S5B). These data supported the likelihood for IPW efficacy in naturally aged mice, since it showed not only excellent target engagement, but also efficacy in reducing symptomatic hyperexcitability induced by iAlb.

\section{Drug inhibition of TGF $\beta$ signaling reverses molecular and functional brain aging in mice}

Based on these validation studies, we then tested IPW as an intervention against TGF $\beta$ signaling in mice aged to 2 years old, near the end of the mouse lifespan. Immunofluorescent analysis of hippocampal sections from aged mice revealed that 5 days of treatment with IPW $(20 \mathrm{mg} / \mathrm{kg}$ i.p.) reduced the number of astrocytes co-labeled with pSmad2 (Fig. 6A). Similarly, hippocampal Western blot showed that 5 days of IPW treatment reduced the high levels of pSmad2 in aged mice, thus restoring a "healthy" level of TGF $\beta$ signaling similar to that of young mice (Fig. 6B). Furthermore, IPW treatment reduced the downstream output TGF $\beta 1$ (Fig. 6C).

To test if IPW inhibition of TGF $\beta$ signaling could reverse the symptoms of neural hyperexcitability in aged mice, we treated 24-month-old mice with IPW for 7 days, and then performed the PTZ assay. As in aTGF $\beta R$ KD genetic intervention, mice treated with IPW showed lower seizure severity and mortality compared to aged control mice treated with vehicle (Fig. 6D; Fig. S6A). To further investigate the efficacy of IPW on aberrant neural activity, we conducted a longitudinal experimental design in which young and old mice from the ECoG cohort were continuously recorded for 5 days of baseline, followed by 5 days of IPW treatment, and then 5 days of "washout" (no further dosing). This design was intended to assess not only the acute efficacy of IPW for treating symptoms of aberrant ECoG activity, but also whether any treatment effects persist after dosing is halted.

During the baseline recording period, we observed that approximately half of the aged mice showed a phenotype of high pSWEs, whereas others showed no aberrant ECoG activity. These subgroups were confirmed by unbiased clustering analysis, which defined a best fit for the aged mouse population as two distinct clusters of high pSWE (h-SWE) and low pSWE (I-SWE) mice (Fig. S6B-D). In the longitudinal experimental design, we thus analyzed these subgroups separately, in order to assess the efficacy of IPW treatment on the h-SWE phenotype. In h-SWE mice, treatment with IPW dramatically reduced the number of pSWEs, restoring a profile of ECoG activity similar to that of young mice (Fig. 6E). In contrast, IPW treatment had no effect on I-SWE mice, or on young mice, which did not show any aberrant high pSWE activity. Furthermore, treatment of aged mice with vehicle control showed no efficacy on reducing pSWEs (Fig. S6E-F). Beyond the treatment phase, the efficacy of IPW on reducing h-SWEs also persisted through the end of the washout period, indicating that inhibition of TGF $\beta$ signaling may mediate a long-lasting change in the underlying hyperexcitability of neural circuits.

Given the effects of IPW on inhibiting TGF $\beta$ signaling and reversing associated outcomes of hyperexcitability and ECoG neural network dysfunction, we next assessed functional cognitive outcomes. Aged mice were treated for 7 days with IPW or vehicle control, and then assessed in two cognitive behavioral tasks performed over consecutive days: spontaneous alternation in T maze, and the novel object task, which is also sensitive to age-related memory decline $(62,63)$. After 7 days of IPW treatment, aged mice showed significant improvement in both cognitive tasks, relative to vehicletreated controls (Fig. 6F-G), demonstrating that IPW is effective in improving cognitive impairment in aged mice. Together, these studies show that IPW inhibition of chronic TGF $\beta$ signaling in aged mice can rapidly restore a "youthful" profile of network activity and cognitive capacity.

\section{Discussion}


Aging is often accompanied by cognitive decline, even in the absence of dementia or measurable neurodegeneration $(64,65)$. Unlike transgenic models for artificially inducing age-like disease, our investigations focused on naturally aging mice, allowing us to observe the relative sequence of biological changes associated with brain aging. We found that BBB dysfunction and consequent albumin extravasation appears as early as middle age, placing it among the earliest known hallmarks of the aging brain. Consistent with our findings, relatively subtle changes in neural and synaptic function have been widely observed in humans and other mammals as one of the first signs of neurological aging (64-67), and these changes in neurotransmission are associated with hippocampal hyperexcitability that is thought to be one of the earliest events in the progression of mild cognitive impairment $(43,44,68)$. However, the regulatory pathways that may trigger or control these changes are unknown. We found that microvascular BBB dysfunction allows for the extravasation of serum albumin into the brain and hyperactivation of TGF $\beta$ signaling, similar to the activation of TGF $\beta$ signaling that has been shown in head injury models (22). Activation of this signaling cascade in turn causes symptoms associated with aberrant neural function (Fig. 7).

We used telemetric ECoG to directly record abnormal neural network activity during aging. We found slowing of EEG/ECoG activity, consistent with other reports in the context of aging (46-48). We further showed that this slowing of activity in mice is characterized as discrete, paroxysmal transient events (pSWEs), occuring frequently and spontaneously against a backdrop of "normal" ECoG activity. These pSWEs, which have characteristics that are similar to but distinct from epileptic seizures, may constitute "silent" or "subclinical" epileptiform activity, as has been reported in human dementia patients (69-71). These data support the suggestion, proposed elsewhere (72-74), that there may be common mechanistic links between age-related dementia and epilepsy, which has a remarkably high incidence in the elderly (75-77). We emphasize that elevated TGF $\beta$ signaling, triggered by BBB dysfunction, provides a simple, parsimonious model for how this dysfunction may arise in aging. Indeed, we found that TGF $\beta$ inhibition reversed aberrant ECoG activity, increased seizure threshold, and improved cognitive outcomes in aged mice, suggesting efficacy in the myriad symptoms that would be expected to arise from a dysfunctional neural network.

Could inhibition of TGF $\beta$ signaling, as a strategy to counteract the detrimental consequences of agerelated BBB dysfunction, hold therapeutic potential? One of the major challenges (and causes of failure) in treating progressive neurological diseases is that patients decline over time, and thus may accumulate irreversible damage by the time of diagnosis. Considering that BBB dysfunction begins relatively early in aging, this might call for a scenario in which chronic preventive treatment with TGF $\beta$ inhibitors is requried to avoid future damage. However, we found that one week of acute treatment reversed the pathological outcomes in aged mice, including elevated TGF $\beta$ signaling, aberrant ECoG activity, seizure vulnerability, and cognitive dysfunction. Our findings suggest that the aging brain may retain considerable cognitive capacity, which may be chronically suppressed (but not irreversibly lost) by BBB leakiness and its inflammatory fallout.

By uncovering a foundational mechanism linking BBB decline to neural dysfunction, our study raises several critical questions: what causes BBB decline itself? And is BBB decline causal to, concurrent with, or an outcome of (or independent from) other well-known mechanisms of aging such as inflammation, reactive oxidation stress and metabolic failures, proteasome senescence, DNA damage, etc. (78-83)? Our work, while novel in focusing on microvascular integrity and interactions within the neurovascular unit, can be placed in context of some of these mechanisms. For example, activation of astrocytes and gliosis has been shown to be a key step in many different aging diseases, with astrocytes playing potent 
roles in controlling neuroinflammation and neural functions including synaptic plasticity, senescence, and neurodegeneration (84-88); we show that BBB dysfunction may be an early step causing or contributing to activation of astrocytes and the ensuing inflammatory response. Similarly, several previous studies have reported increased brain TGF $\beta$ signaling in aging (89-94), and suggested that it could be a primary regulatory factor inducing aged neural phenotypes - although it was unknown what may trigger this increase in TGF $\beta$ signaling in aged individuals. We show that extravasation of albumin through the leaky BBB and astrocytic uptake may be one of the earliest steps that induces this agerelated TGF $\beta$ cascade. Ultimately, our mechanistic findings provide a guiding framework for translation into the human clinical context, including large-scale epidemiological studies that are needed to establish the relationship between BBB status, other known biomarkers, and disease outcomes. In particular, this research offers new hope in two key unmet areas: early detection (via MRI imaging of BBB status), and a new avenue for disease-modifying treatment that is mechanistically distinct from other canonical dementia targets, many of which have failed in clinical trials.

\section{Materials and Methods}

Study design and statistical analysis. The aim of this study was to use experimental gain-of-function (iAlb) and loss-of-function (TGF $\beta R$ KD and IPW) interventions to investigate the causal role of TGF $\beta$ signaling in age-relate BBB pathology and the efficacy of therapeutic intervention. Outcomes were assessed using molecular (immunofluorescent staining and WB), electrophysiological (ECoG), and behavioral (PTZ and cognitive tasks) measures of symtomatic pathology in rodents. Translatability of these findings was further supported by complementary measures of BBB permeability and TGF $\beta$ signaling in human subjects (via CTE-MRI imaging and post-mortem histology). The experimental designs and methodology of outcome measures were chosen prior to initiating each experiment, except in the case of the ECoG experiments, in which post-hoc analysis of ECoG signal led to the discovery of pSWEs as a novel biomarker of aberrant network activity. For all experiments, subjects were randomly assigned to experimental groups, and data were collected under blinded experimental conditions with the exception of the immunofluorescent albumin counts in Fig. 1A, in which differences between age groups were visually apparent in the tissue even under naïve conditions, such that complete blinding was not possible. The lead and corresponding authors were responsible for experiment design, conducting statistical analysis, and unblinding the final results. All graphs are plotted showing mean and SE. Two sample comparisons were performed by student's t-test or Mann-Whitney test, and multiple group comparisons were conducted by ANOVA or Kruskal-Wallis test, followed by post-hoc testing to compare individual groups when a main effect was detected. Multiple correction comparisons were used as described in figure legends. Seizure progression in the PTZ experiments was analyzed by two-way ANOVA, and linear regression was used to calculate regression slopes. Differences in regression slope were also compared by ANOVA. In mouse ECoG experiments, different subgroups (h-SWE and I-SWE) were observed after data collection. These subgroups were formally classified using an unbiased Gaussian mixed model, and then inferential statistics were performed on the subsequent groups. For all inferential statistics, two-tailed tests were used and significance thresholds were set at $p<0.05$. For rodent behavior experiments, animals were removed from the study cohort if they were unable to complete the requisite task, according to the following pre-defined criteria: for MWM, mouse shows inability to swim (unable to maintain swim speed or buoyancy); for T-maze, mouse does not leave stem to complete arm choice in greater than three trials. For TGF 1 WB analysis following IPW treatment (Fig. $6 C)$, two samples were excluded due to gel loading error. Exclusion of these data did not alter statistical significance (results of ANOVA analysis were significant with or without excluded values). 
BBB imaging. The human imaging protocol was approved by the Soroka University Medical Center Helsinki institutional review board, and written informed consent was given by all participants. BBB status was assessed by DCE-MRI in $n=105$ subjects with an age range of 21 to 83 years old. MRI scans were performed using a 3T Philips Ingenia scanner, and included: T1-weighted anatomical scan (3D gradient echo, TE/TR = 3.7/8.2 ms, acquisition matrix $432 \times 432$, voxel size: $0.5 \times 0.5 \times 1 \mathrm{~mm}$ ), T2-weighted imaging (TE/TR $=90 / 3000 \mathrm{~ms}$, voxel size $0.45 \times 0.45 \times 4 \mathrm{~mm}$ ). For the calculation of pre-contrast longitudinal relaxation times ( $T_{10}$ ), variable flip angle (VFA) method was used (3D T1w-FFE, TE/TR = 2/10 ms, acquisition matrix: $256 \times 256$, voxel size: $0.89 \times 0.89 \times 6 \mathrm{~mm}$, flip angles: 10, 15, 20, 25 and $30^{\circ}$ ). Dynamic contrast-enhanced (DCE) sequence was then acquired (Axial, 3D T1w-FFE, TE/TR = 2/4 ms, acquisition matrix: $192 \times 187$ (reconstructed to $256 \times 256$ ), voxel size: $0.9 \times 0.9 \times 6 \mathrm{~mm}$, flip angle: $20^{\circ}, \Delta \mathrm{t}=10$ Sec, temporal repetitions: 100, total scan length: 16.7 minutes). An intravenous bolus injection of gadoterate meglumine (Gd-DOTA, Dotarem, Guerbet, France) was administered using an automatic injector after the first five DCE repetitions. Data preprocessing included image registration and normalization to MNI coordinates (using SPM (http://www.fil.ion.ucl. ac.uk/spm)). BBB permeability was calculated for each brain voxel using in-house MATLAB script (Mathworks, USA), as described(95-97). Briefly, a linear fit is applied to the later part of the concentration curve of each voxel; the slope is then divided by the slope at the superior sagittal sinus, to compensate for physiological (e.g., heart rate, blood flow) and technical (e.g., contrast agent injection rate) variability. For region specific permeability, disrupted region voxels were divided by total region voxels according to SPM registration.

Immunostaining. For human staining, postmortem hippocampus was obtained from young $(n=3$, mean age $=31.3 \pm 5$ years) and old patients ( $n=10$, mean age $=70.6 \pm 5.6$ years). All participants gave informed and written consent and all procedures were conducted in accordance with the Declaration of Helsinki and approved by the University of Bonn ethics committee. Resected hippocampi were fixed in $4 \%$ formalin and processed into liquid paraffin. All specimens were sliced at $4 \mu \mathrm{m}$ with a microtome (Microm, Heidelberg, Germany), mounted on slides, dried, and deparaffined in descending alcohol concentration. For mouse samples, mice were anesthetized with Euthasol euthanasia solution and transcardially perfused with ice cold heparinized physiological saline (10 units heparin/mL physiological saline) followed by $4 \%$ paraformaldehyde (PFA, Fisher Scientific \#AC416785000) in $0.1 \mathrm{M}$ phosphate buffered saline (PBS). Brains were removed, post-fixed in 4\% PFA for 24 hours at $4^{\circ} \mathrm{C}$, and cryoprotected in $30 \%$ sucrose in PBS. Brains were then embedded in Tissue-Tek O.C.T. compound (Sakura, Torrance, CA), frozen, and sliced on a cryostat into $20 \mu \mathrm{m}$ coronal sections, mounted on slides. Samples were immunostained under the following protocol. Slides were treated for antigen retrieval (for human, $5 \mathrm{~min}$ incubation at $100^{\circ} \mathrm{C}$ in sodium citrate buffer, $\mathrm{pH}$ 6.0); for mouse, $15 \mathrm{~min}$ incubation at $65^{\circ} \mathrm{C}$ in Tris-EDTA buffer (10mM Tris Base, $1 \mathrm{mM}$ EDTA solution, 0.05\% Tween 20, pH 9.0), then incubated in blocking solution ( $5 \%$ Normal Donkey Serum in $0.1 \%$ Triton X-100/TBS) for 1 hour at room temperature. Samples were then stained with primary antibody at $4^{\circ} \mathrm{C}$, followed by fluorescent-conjugated secondary antibody for 1 hour at room temperature, and then incubated with DAPI (900 nM; Sigma-Aldrich) to label nuclei. For human, primary antibodies were rabbit anti-phosphorylated Smad2 (Millipore AB3849-I, 1:500), chicken anti-Albumin (Abcam ab106582, 1:500), and mouse anti-GFAP (Millipore MAB3402, 1:500); for mouse, the same were used except anti-phosphorylated Smad2 (Millipore AB3849) and goat anti-GFAP (Abcam ab53554, 1:1000). Secondary antibodies were anti-rabbit Alexa Fluor 568, antichicken Alexa Fluor 647, anti-goat Alexa Fluor 488, anti-mouse Alexa Fluor 488 (1:500, Jackson ImmunoResearch), and anti-goat Alexa Fluor 647 (Abcam ab150131, 1:500). All antibodies dilutions were in blocking solution. For tissue from human patients and aged mice, slide-mounted brain sections for treated with TrueBlack Lipofuscin Autofluorescence Quencher (Biotium \#23007) before coverslip mounting. Images were acquired at 20X or 40X objective magnification using a Zeiss Axio Observer Research microscope (Carl Zeiss AG) and Metamorph software (version 7.7.7.0), and analyzed using 
ImageJ software (NIH). For human samples, counts were performed in 10 of randomly selected sampling areas per subject. For mouse, imaging was performed in at least 3-4 hippocampi per mouse. Cell counts for each individual marker, and colabeling, were calculated manually by an observer, and normalized to the area or total number of DAPI-positive cells. For each subject, counts from each sampling area were averaged.

Human transcriptome analysis. To investigate TGF $\beta$ gene expression patterns in brain hippocampus we evaluated expression data of 123 individuals from the Genotype-Tissue Expression (GTEx) project v7 release (56). RNA sequencing was done by the GTEx consortium to measure the gene expression level from samples, reported as transcripts per million (TPM). We obtained data derived from hippocampal tissue samples for expression of the TGF $\beta$ isoforms TGF $\beta 1$ (ENSG00000105329.5), TGF $\beta 2$ (ENSG00000092969.7), and TGFß3 (ENSG00000119699.3), and performed linear regression and Pearson correlations on the resulting datasets with respect to subject age.

Evans Blue assay. $4 \%$ Evans Blue (EB) solution in $0.9 \%$ sterile saline was administered by i.v. bolus injection through the tail vein at a dose of $2 \mathrm{~mL} / \mathrm{kg}$. Thirty minutes after tracer administration, brains were transcardially perfused at a rate of $2 \mathrm{~mL} / \mathrm{min}$ first with PBS for 5 minutes, then with $4 \%$ PFA dissolved in PBS for 10 minutes. The brain was removed, post-fixed, and cryoprotected in $30 \%$ sucrose in PBS. Brains were cryosectioned at 20 um and slide mounted using Entellan mounting solution (Millipore). Sections were imaged at 20X objective magnification using an Axio Scan.Z1 slide scanner (Zeiss), and quantified blindly to determine the number of EB positive cells within the hilar dentate gyrus of the hippocampus.

RT-qPCR. Total RNA was extracted from 200-250 mg of frozen mouse hippocampal tissue using TRIzol reagent (ThermoFisher Scientific \#15596026), and further purified with DNA-free DNA Removal Kit (ThermoFisher Scientific AM1906). First-strand cDNA synthesis was performed from $1 \mu \mathrm{g}$ isolated RNA template using iScript RT supermix (Bio-Rad \#1708841). PCR products were amplified using a CFX96 Real-Time PCR System (Bio-Rad), and threshold cycles were detected using SsoAdvanced Universal SYBR Green Supermix (Bio-Rad \#172-5271). Mean threshold cycles were normalized to 18s or GAPDH internal control, and relative gene expression levels were quantified using the 2- $\Delta \Delta \mathrm{CT}$ method. Primer sequences were obtained from the $\mathrm{NCl} / \mathrm{NIH}$ qPrimerDepot and are listed in Table S6.

Western Blot. Mouse or rat hippocampal tissue was homogenized and protein lysates were extracted using RIPA buffer (50 mM Tris- $\mathrm{HCl}, 150 \mathrm{mM} \mathrm{NaCl}, 1 \% \mathrm{NP}-40,0.5 \%$ Sodium deoxycholate, $0.1 \%$ SDS) including a protease (Calbiochem \#539134) and phosphatase inhibitor cocktail (Roche PhoStop Ref: 4906845001). Protein samples were run under reducing conditions. $20 \mu \mathrm{g}$ of protein lysate was mixed with Laemmli buffer (Bio-Rad \#161-0737), containing 5\% 2-mercaptoethanol (Sigma M6250), and fractionated by SDS-PAGE using the Mini-PROTEAN Tetra System and pre-cast TGX'M Gels (Bio-Rad \#4561096); Following separation, samples were transferred to a nitrocellulose membrane $(0.45 \mu \mathrm{m}$, Bio-Rad \#1620115). Membranes were blocked for $1 \mathrm{hr}$ at room temperature with 5\% non-fat dry milk (Apex \#20241) or 5\% BSA (Biotium \#22013) in TBST (10 mM Tris, $150 \mathrm{mM} \mathrm{NaCl}, 0.5 \%$ Tween 20, pH 8.0), and incubated overnight at $4{ }^{\circ} \mathrm{C}$ with primary antibody. Membranes were then washed $3 \times 10$ min with TBST and incubated with secondary antibodies for $1 \mathrm{hr}$ at room temperature. Membranes were washed with TBST 3x10min and visualized using chemiluminescence SuperSignal West Dura Extended Substrate (ThermoFisher Scientific \#34075), and Bio-Rad Chemidoc system with Bio-Rad Image Lab software (version 4.0.1). Densitometry analysis was done using Image J (NIH). The following primary and secondary antibodies were used: rabbit anti- $\beta$-Actin (1:2000, Cell Signaling \#4970), rabbit anti-GAPDH (1:2000, Cell Signaling \#2118), rabbit anti-TGFßR2 (1:1500, Abcam ab186838), rabbit anti- 
phosphorylated Smad2 (1:1000, Millipore AB3849-I), rabbit anti-phosphorylated Smad2 (1:1000, Millipore AB3849), anti-rabbit TGFß1 (1:500, ab92486), anti-rabbit HRP (1:2000, Cell Signaling \#4970).

Osmotic pump implants. For mice, pumps were implanted as previously described(98). Briefly, surgery was performed on adult male mice under isofluorane anesthesia (2\%). Using a stereotaxic frame, a hole was drilled through the skull at $0.5 \mathrm{~mm}$ posterior, $1 \mathrm{~mm}$ lateral to bregma, and a cannula was placed into the right lateral cerebral ventricle, fixed with surgical glue. Cannulas (Brain infusion kit BIK 3, \#0008851, Alzet, Cupertino, CA) were attached to micro-osmotic pumps (Model 2001, ALZET) filled with $200 \mu \mathrm{L}$ of either $0.4 \mathrm{mM}$ bovine serum albumin (Alb; Sigma-Aldrich) solution or artificial cerebrospinal fluid (aCSF) as previously described (99), and implanted subcutaneously in the right flank. In a subset of animals, $10 \%$ of the Alb was replaced with Alexa Fluor 647 conjugated BSA ( $2.68 \mathrm{~g} / \mathrm{L}$; ThermoFisher Scientific A34785). Pumps infused at a rate of $1.0 \mu \mathrm{L} / \mathrm{hr}$ for the duration described in each experiment. For rats, 10 week-old male Wistar rats were used, and surgeries were performed the same way, using the following coordinates: $-1 \mathrm{~mm}$ posterior and $1.5 \mathrm{~mm}$ lateral to bregma. For rats, albumin was used at a concentration of $0.2 \mathrm{mM}$ and infused for 7 days at a rate of $10 \mu \mathrm{L} / \mathrm{hr}$ via larger micro-osmotic pumps (Model 2ML1, Alzet).

ECoG. ECoG was recorded as previously reported (49) from 9- to 12-wk-old Wistar male rats implanted with osmotic pumps, and from young ( 3 months, $n=5)$ and old (18-22 months, $n=20)$ mice. In brief, under stereotaxic surgery and $2 \%$ isoflurane anesthesia, two screw electrodes were implanted in each hemisphere (rat coordinates: $4.8 \mathrm{~mm}$ posterior, $2.7 \mathrm{~mm}$ anterior, and $2.2 \mathrm{~mm}$ lateral; mouse coordinates: 0.5 and $3.5 \mathrm{~mm}$ posterior and $1 \mathrm{~mm}$ lateral, all relative to bregma). A wireless transmitter (Data Science International, Saint Paul, MN, US) was placed in a dorsal subcutaneous pocket, and leads connected to the screws. Connections were isolated and fixed by bone cement such that one ECoG channel was associated with each hemisphere. For rat surgeries, a cannula (-1 mm caudal, 1.5 lateral, 4 $\mathrm{mm}$ depth) and osmotic pump (infusing at $2.4 \mu \mathrm{l} / \mathrm{h}$ ) were also implanted (28). Animals were treated with post-operative buprenorphine $(0.1 \mathrm{mg} / \mathrm{Kg})$ and allowed to recover for at least $72 \mathrm{hrs}$ prior to recording. Continuous, bichannel ECoG (sampling rate, $500 \mathrm{~Hz}$ ) was recorded wirelessly from freely roaming animals in the home cage for the duration of experiments described. Rats were recorded during the first and the fourth week following surgery. Mice were recorded for 15 days, starting 1 week after surgery, and administered IPW ( $20 \mathrm{mg} / \mathrm{kg}$ ) on days $6-10$ of recording. To detect PSWE, ECoG signals were buffered into $2 \mathrm{sec}$ long epochs with an overlap of $1 \mathrm{sec}$. Fast Fourier Transform (FFT) was applied and the frequency of median power was extracted for each epoch. Thus, an event was considered as a pSWE if its frequency of median power was less than $5 \mathrm{~Hz}$ for 10 consecutive seconds or more. FFT was also applied for the entire recording period to analyze relative power across the frequency spectrum of 1-20 $\mathrm{Hz}$.

Animal care and transgenic mice. All animal procedures were approved by the institutional animal care committees. Animals were housed with a 12:12 light:dark cycle with food and water available ad libitum. Aldh1L1-eGFP mice were bred from STOCK Tg(Aldh1l1-EGFP)OFC789Gsat/Mmucd (identification number 011015-UCD), purchased from the Mutant Mouse Regional Resource Center. These FVB/N mice were crossed to a $\mathrm{C} 57 \mathrm{BL} / 6$ genetic background. The resulting strain exhibits constitutive astrocytic expression of eGFP protein under the astrocytic promoter Aldh1L1.

Triple transgenic aTGF $\beta R / K D$ mice were bred from strains purchased from the Jackson Laboratory to generate mice that express CreERT under the astrocytic promoter glial high affinity glutamate transporter (GLAST), with a floxed exon 4 of TGF- $\beta$ R2 (tgfbr $\left.2^{\mathrm{fl}}\right)$, and a transgenic LacZ reporter gene inhibited by a floxed neomycin cassette. Tamoxifen induction thus induces activation of astrocytic 
CreERT resulting in a null TGFßR2 allele (tgfbr2null) and LacZ expression (R26 $\mathrm{R}^{-/-}$). The parental strain STOCK Tg(Slc1a3-cre/ERT)1Nat/J mice were outcrossed with B6;129-Tgfbr2tm1Karl/J and B6.129S4Gt(ROSA)26Sortm1Sor/J mice to produce males, while B6;129-Tgfbr2tm1Karl/J and B6.129S4Gt(ROSA)26Sortm1Sor/J mice were outcrossed to produce females. The resulting GLAST-CreERT; tgfbr2 ${ }^{\mathrm{fl} /+}$ males were bred with tgfbr2fl/+; R26R-/- and tgfbr2 ${ }^{\mathrm{fl} / \mathrm{fl}} ; \mathrm{R}^{2} 2 \mathrm{R}^{-/-}$females to produce triple transgenic offspring. Subsequent generations were incrossed to produce experimental triple transgenic

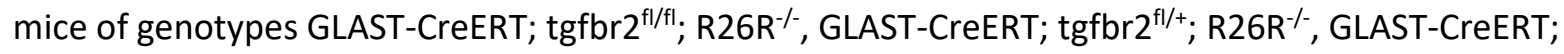

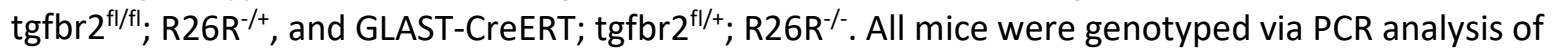
tissue biopsy samples (Table S4).

The inducible Cre/lox system was activated by 5 days of tamoxifen injection (Sigma-Aldrich, $160 \mathrm{mg} / \mathrm{kg}$ dissolved in corn oil, i.p.). Control GLAST-CreERT; tgfbr2 $2^{\mathrm{fl} /+}$ heterozygotes received the same dosage of tamoxifen and control GLAST-CreERT; tgfbr $2^{\mathrm{fl} / \mathrm{fl}}$ mice received i.p. injection of corn oil vehicle at equivalent volumes. Mice were weighed daily to ensure accurate dosage.

Seizure induction. Seizures were induced by a single injection of pentylenetetrazole (PTZ; Sigma, P6500, $85 \mathrm{mg} / \mathrm{kg}$ s.c.). Video recordings were taken for 30 minutes following induction, which were then scored by a blind observer, using a modified Racine scale to quantify progression of seizure severity, as follows: 0 - No Seizures; 1 - Immobility; 2 - Straub's tail; 3 - Forelimb Clonus; 4 - Generalized Clonus; 5 Bouncing Seizure; 6 - Status Epilepticus. Latency to first appearance of each Racine stage was quantified, as was latency to mortality (when occurring prior to the $30 \mathrm{~min}$ endpoint).

Behavior assessments. Spatial working memory was tested by spontaneous alternation in a T-maze constructed of black plastic, with stem $(50 \times 16 \mathrm{~cm})$ and two arms $(50 \times 10 \mathrm{~cm})$. A vertical divider was placed at the midline of the stem exit, thus creating two entryways leading to either the right or left arms. Naiive mice were placed at the beginning of the stem and allowed to freely roam until opting to enter either the right or left arm. Upon crossing the threshold of the chosen arm, a door was lowered, and the mouse was contained to the chosen arm for $30 \mathrm{sec}$. Then, the mouse was returned to the stem and the door raised, allowing the next choice trial to commence immediately. After 10 sequential trials, the mouse was returned to the home cage. "Correct" alternation choices were scored when the mouse chose the arm opposite of that chosen in the previous trial, and percent correct was calculated as (total correct choices) / (total number of completed trials). In the event that a mouse did not leave the stem within $60 \mathrm{sec}$, it was removed from the apparatus for $30 \mathrm{sec}$, and then reset in the stem to start a new trial. These "incomplete" trials were not counted in scoring. Spatial memory in young mice was assessed in the Morris Water Maze (MWM) task (100). Because of excess colony availability, heterozygous control mice from the transgenic colony (TGF $\beta 22(\mathrm{fl} /+)$ genotype), which retain normal TGF $\beta$ signaling, were used for the MWM study cohort. For each trial, mice were placed at randomized starting locations in a MWM pool filled with opaque water (colored with non-toxic white acrylic paint), with visual cues placed on the pool walls. Mice were allowed to swim freely until locating a hidden platform under the surface of the water (or guided to the platform after 40 seconds of failed searching), and then left on the platform for 10 seconds prior to starting a new trial. Spatial learning was quantified by measuring latency to reach the platform, averaged from four trials per day, with training over 9 consecutive days of learning. Object memory was tested in the novel object task, using a 3-day protocol consisting of a 10 min trial on each day, recorded by overhead video. On day 1 mice were habituated to a square testing chamber, constructed of white plastic $(50 \times 50 \mathrm{~cm})$, with two unfamiliar objects placed inside. On day 2, the previous objects were removed and 3 new objects were placed in a " $L$ " configuration, equidistant from each other and the walls. On day 3, one of the objects was removed and replaced by a new object, thus leaving 2 familiar objects and 1 novel object. A blind observer quantified duration of time spent 
investigating each object (scoring criteria were mouse nose oriented towards object at a distance of 1 $\mathrm{cm}$ or less), and percentage of time at the novel object was quantified as (duration investigating novel object) / (total duration investigating all three objects). The set objects were chosen from common items such as lab bottles, pipette boxes, cups (placed open-side down), etc., that were of similar dimensions but varied in shape, color, and material. The sequence and position of objects used across trials was identical for all mice.

IPW pharmacokinetics. Brain concentration measurement of IPW-5371 was performed by Jubilant Biosys. The experiment was approved by the Jubilant Biosys Institutional Animal Ethics Committee, Bangalore, India (IAEC/JDC/2015/72) and were in accordance with the Committee for the Purpose of Control and Supervision of Experiments on Animals (CPCSEA), Ministry of Social Justice and Environment, Government of India. Twelve Balb/C mice (age 6-7 weeks) were procured from Bioneeds, Bangalore, India. Animals were housed in Jubilant Biosys animal care facility in a temperature and humidity controlled room with a 12:12 h light:dark cycles, had free access to food (Provimin, India) and water for one week before experimental use. Following $\sim 4 \mathrm{~h}$ fasting (during fasting period animals had free access to water) mice received IPW- 5371 orally at a dose of $20 \mathrm{mg} / \mathrm{kg}$ (dissolved in $0.5 \%$ methylcellulose and saline). Mice were euthanized under isofluorane and the brain was removed and weighed. Brain tissue homogenates were prepared with $10 \%$ tetrahydrofuran in acetonitrile (tissue was homogenated with $4 \mathrm{~mL} / \mathrm{g}$ of $10 \%$ tetradhydrofuran in acetonitrile containing IS $(100 \mathrm{ng} / \mathrm{mL}))$. Subsequently, $250 \mu \mathrm{L}$ of brain homogenate was centrifuged at $14,000 \mathrm{rpm}$ for $5 \mathrm{~min}$ at $10^{\circ} \mathrm{C}$. An aliquot of $10 \mu \mathrm{L}$ was injected onto an API 4000 LC-MS/MS system for analysis.

\section{References}

1. Z. Zhao, A. R. Nelson, C. Betsholtz, B. V. Zlokovic, Establishment and Dysfunction of the Blood-Brain Barrier, Cell 163, 1064-1078 (2015).

2. N. J. Abbott, A. A. K. Patabendige, D. E. M. Dolman, S. R. Yusof, D. J. Begley, Structure and function of the blood-brain barrierNeurobiol. Dis. 37, 13-25 (2010).

3. G. Tibbling, H. Link, S. Ohman, Principles of albumin and IgG analyses in neurological disorders. I. Establishment of reference values., Scand. J. Clin. Lab. Invest. 37, 385-90 (1977).

4. B. V. Zlokovic, Neurovascular pathways to neurodegeneration in Alzheimer's disease and other disorders, Nat. Rev. Neurosci. (2011), doi:10.1038/nrn3114.

5. N. Zeevi, J. Pachter, L. D. McCullough, L. Wolfson, G. A. Kuchel, The blood-brain barrier: geriatric relevance of a critical brain-body interface., J. Am. Geriatr. Soc. 58, 1749-57 (2010).

6. G. A. Rosenberg, Blood-Brain Barrier Permeability in Aging and Alzheimer's Disease., J. Prev. Alzheimer's Dis. 1, 138-139 (2014).

7. M. D. Sweeney, A. P. Sagare, B. V. Zlokovic, Blood-brain barrier breakdown in Alzheimer disease and other neurodegenerative disorders, Nat. Rev. Neurol. (2018), doi:10.1038/nrneurol.2017.188.

8. A. J. Farrall, J. M. Wardlaw, Blood-brain barrier: ageing and microvascular disease--systematic review and meta-analysis., Neurobiol. Aging 30, 337-52 (2009).

9. E. Zenaro, G. Piacentino, G. Constantin, The blood-brain barrier in Alzheimer's disease, Neurobiol. Dis. 107, 41-56 (2017). 
10. T. Skillbäck, L. Delsing, J. Synnergren, N. Mattsson, S. Janelidze, K. Nägga, L. Kilander, R. Hicks, A. Wimo, B. Winblad, O. Hansson, K. Blennow, M. Eriksdotter, H. Zetterberg, CSF/serum albumin ratio in dementias: a cross-sectional study on 1861 patients., Neurobiol. Aging 59, 1-9 (2017).

11. H. J. van de Haar, S. Burgmans, J. F. A. Jansen, M. J. P. van Osch, M. A. van Buchem, M. Muller, P. A. M. Hofman, F. R. J. Verhey, W. H. Backes, Blood-Brain Barrier Leakage in Patients with Early Alzheimer Disease, Radiology 281, 527-535 (2016).

12. D. A. Nation, M. D. Sweeney, A. Montagne, A. P. Sagare, L. M. D'Orazio, M. Pachicano, F. Sepehrband, A. R. Nelson, D. P. Buennagel, M. G. Harrington, T. L. S. Benzinger, A. M. Fagan, J. M. Ringman, L. S. Schneider, J. C. Morris, H. C. Chui, M. Law, A. W. Toga, B. V. Zlokovic, Blood-brain barrier breakdown is an early biomarker of human cognitive dysfunction, Nat. Med. (2019), doi:10.1038/s41591-018-0297-y.

13. A. Montagne, S. R. Barnes, M. D. Sweeney, M. R. Halliday, A. P. Sagare, Z. Zhao, A. W. Toga, R. E. Jacobs, C. Y. Liu, L. Amezcua, M. G. Harrington, H. C. Chui, M. Law, B. V. Zlokovic, Blood-Brain Barrier Breakdown in the Aging Human Hippocampus, Neuron 85, 296-302 (2015).

14. N. Bien-Ly, C. A. Boswell, S. Jeet, T. G. Beach, K. Hoyte, W. Luk, V. Shihadeh, S. Ulufatu, O. Foreman, Y. Lu, J. DeVoss, M. van der Brug, R. J. Watts, Lack of Widespread BBB Disruption in Alzheimer's Disease Models: Focus on Therapeutic Antibodies, Neuron 88, 289-297 (2015).

15. R. Raja, G. A. Rosenberg, A. Caprihan, MRI measurements of Blood-Brain Barrier function in dementia: A review of recent studiesNeuropharmacology 134, 259-271 (2018).

16. C. S. Janota, D. Brites, C. A. Lemere, M. A. Brito, Glio-vascular changes during ageing in wild-type and Alzheimer's disease-like APP/PS1 mice, Brain Res. 1620, 153-168 (2015).

17. T. J. Montine, W. J. Koroshetz, D. Babcock, D. W. Dickson, W. R. Galpern, M. M. Glymour, S. M. Greenberg, M. L. Hutton, D. S. Knopman, A. N. Kuzmichev, J. J. Manly, K. S. Marder, B. L. Miller, C. H. Phelps, W. W. Seeley, B.-A. Sieber, N. B. Silverberg, M. Sutherland, C. L. Torborg, S. P. Waddy, B. V Zlokovic, R. A. Corriveau, ADRD 2013 Conference Organizing Committee, Recommendations of the Alzheimer's disease-related dementias conference., Neurology 83, 851-60 (2014).

18. O. Tomkins, A. Feintuch, M. Benifla, A. Cohen, A. Friedman, I. Shelef, Blood-brain barrier breakdown following traumatic brain injury: a possible role in posttraumatic epilepsy., Cardiovasc. Psychiatry Neurol. 2011, 765923 (2011).

19. J. R. Hay, V. E. Johnson, A. M. H. Young, D. H. Smith, W. Stewart, Blood-Brain Barrier Disruption Is an Early Event That May Persist for Many Years After Traumatic Brain Injury in Humans, J. Neuropathol. Exp. Neurol. 74, 1147-1157 (2015).

20. C. A. Tagge, A. M. Fisher, O. V Minaeva, A. Gaudreau-Balderrama, J. A. Moncaster, X.-L. Zhang, M. W. Wojnarowicz, N. Casey, H. Lu, O. N. Kokiko-Cochran, S. Saman, M. Ericsson, K. D. Onos, R. Veksler, V. V Senatorov, A. Kondo, X. Z. Zhou, O. Miry, L. R. Vose, K. R. Gopaul, C. Upreti, C. J. Nowinski, R. C. Cantu, V. E. Alvarez, A. M. Hildebrandt, E. S. Franz, J. Konrad, J. A. Hamilton, N. Hua, Y. Tripodis, A. T. Anderson, G. R. Howell, D. Kaufer, G. F. Hall, K. P. Lu, R. M. Ransohoff, R. O. Cleveland, N. W. Kowall, T. D. Stein, B. T. Lamb, B. R. Huber, W. C. Moss, A. Friedman, P. K. Stanton, A. C. McKee, L. E. Goldstein, Concussion, microvascular injury, and early tauopathy in young athletes after impact head injury and an impact concussion mouse model., Brain 141, 422-458 (2018).

21. C. Schachtrup, J. K. Ryu, M. J. Helmrick, E. Vagena, D. K. Galanakis, J. L. Degen, R. U. Margolis, K. 
Akassoglou, Fibrinogen Triggers Astrocyte Scar Formation by Promoting the Availability of Active TGF- after Vascular Damage, J. Neurosci. 30, 5843-5854 (2010).

22. L. P. Cacheaux, S. Ivens, Y. David, A. J. Lakhter, G. Bar-Klein, M. Shapira, U. Heinemann, A. Friedman, D. Kaufer, Transcriptome profiling reveals TGF-beta signaling involvement in epileptogenesis., $J$. Neurosci. 29, 8927-35 (2009).

23. S. Ivens, D. Kaufer, L. P. Flores, I. Bechmann, D. Zumsteg, O. Tomkins, E. Seiffert, U. Heinemann, A. Friedman, TGF-beta receptor-mediated albumin uptake into astrocytes is involved in neocortical epileptogenesis., Brain 130, 535-47 (2007).

24. G. Bar-Klein, L. P. Cacheaux, L. Kamintsky, O. Prager, I. Weissberg, K. Schoknecht, P. Cheng, S. Y. Kim, L. Wood, U. Heinemann, D. Kaufer, A. Friedman, Losartan prevents acquired epilepsy via TGF- $\beta$ signaling suppression., Ann. Neurol. 75, 864-75 (2014).

25. N. Levy, D. Z. Milikovsky, G. Baranauskas, E. Vinogradov, Y. David, M. Ketzef, S. Abutbul, I. Weissberg, L. Kamintsky, I. Fleidervish, A. Friedman, A. Monsonego, Differential TGF- $\beta$ Signaling in Glial Subsets Underlies IL-6-Mediated Epileptogenesis in Mice., J. Immunol. 195, 1713-22 (2015).

26. S. Y. Kim, V. V Senatorov, C. S. Morrissey, K. Lippmann, O. Vazquez, D. Z. Milikovsky, F. Gu, I. Parada, D. A. Prince, A. J. Becker, U. Heinemann, A. Friedman, D. Kaufer, TGF $\beta$ signaling is associated with changes in inflammatory gene expression and perineuronal net degradation around inhibitory neurons following various neurological insults., Sci. Rep. 7, 7711 (2017).

27. S. Salar, E. Lapilover, J. Müller, J.-O. Hollnagel, K. Lippmann, A. Friedman, U. Heinemann, Synaptic plasticity in area CA1 of rat hippocampal slices following intraventricular application of albumin., Neurobiol. Dis. 91, 155-65 (2016).

28. I. Weissberg, L. Wood, L. Kamintsky, O. Vazquez, D. Z. Milikovsky, A. Alexander, H. Oppenheim, C. Ardizzone, A. Becker, F. Frigerio, A. Vezzani, M. S. Buckwalter, J. Huguenard, A. Friedman, D. Kaufer, Albumin induces excitatory synaptogenesis through astrocytic TGF- $\beta / A L K 5$ signaling in a model of acquired epilepsy following blood-brain barrier dysfunction., Neurobiol. Dis. 78, 115-25 (2015).

29. D. S. Reynolds, A. J. Morton, Changes in blood-brain barrier permeability following neurotoxic lesions of rat brain can be visualised with trypan blue., J. Neurosci. Methods 79, 115-21 (1998).

30. B. Olsson, R. Lautner, U. Andreasson, A. Öhrfelt, E. Portelius, M. Bjerke, M. Hölttä, C. Rosén, C. Olsson, G. Strobel, E. Wu, K. Dakin, M. Petzold, K. Blennow, H. Zetterberg, CSF and blood biomarkers for the diagnosis of Alzheimer's disease: a systematic review and meta-analysis, Lancet Neurol. 15, 673-684 (2016).

31. C. Nordborg, T. E. Sokrab, B. B. Johansson, The relationship between plasma protein extravasation and remote tissue changes after experimental brain infarction., Acta Neuropathol. 82, 118-26 (1991).

32. M. E. Bach, M. Barad, H. Son, M. Zhuo, Y. F. Lu, R. Shih, I. Mansuy, R. D. Hawkins, E. R. Kandel, Agerelated defects in spatial memory are correlated with defects in the late phase of hippocampal longterm potentiation in vitro and are attenuated by drugs that enhance the cAMP signaling pathway., Proc. Natl. Acad. Sci. U. S. A. 96, 5280-5 (1999).

33. R. L. Dean, J. Scozzafava, J. A. Goas, B. Regan, B. Beer, R. T. Bartus, Age-related differences in behavior across the life span of the C57BL/6J mouse., Exp. Aging Res. 7, 427-51 (1981).

34. A. J. Gower, Y. Lamberty, The aged mouse as a model of cognitive decline with special emphasis on 
studies in NMRI mice., Behav. Brain Res. 57, 163-73 (1993).

35. C. C. Kaczorowski, J. F. Disterhoft, Memory deficits are associated with impaired ability to modulate neuronal excitability in middle-aged mice., Learn. Mem. 16, 362-6 (2009).

36. H. Shoji, K. Takao, S. Hattori, T. Miyakawa, Age-related changes in behavior in C57BL/6J mice from young adulthood to middle age., Mol. Brain 9, 11 (2016).

37. Y. V Gorina, Y. K. Komleva, O. L. Lopatina, V. V Volkova, A. I. Chernykh, A. A. Shabalova, A. A. Semenchukov, R. Y. Olovyannikova, A. B. Salmina, [The battery of tests for behavioral phenotyping of aging animals in the experiment], Adv Gerontol 30, 49-55 (2017).

38. B. T. Hawkins, R. D. Egleton, Fluorescence imaging of blood-brain barrier disruption, J. Neurosci. Methods 151, 262-267 (2006).

39. Y. David, L. P. Cacheaux, S. Ivens, E. Lapilover, U. Heinemann, D. Kaufer, A. Friedman, Astrocytic dysfunction in epileptogenesis: consequence of altered potassium and glutamate homeostasis?, J. Neurosci. 29, 10588-99 (2009).

40. J. D. Cahoy, B. Emery, A. Kaushal, L. C. Foo, J. L. Zamanian, K. S. Christopherson, Y. Xing, J. L. Lubischer, P. A. Krieg, S. A. Krupenko, W. J. Thompson, B. A. Barres, A Transcriptome Database for Astrocytes, Neurons, and Oligodendrocytes: A New Resource for Understanding Brain Development and Function, J. Neurosci. 28, 264-278 (2008).

41. L.-J. Chew, C. A. DeBoy, V. V. Senatorov, Finding degrees of separation: Experimental approaches for astroglial and oligodendroglial cell isolation and genetic targeting, J. Neurosci. Methods 236, 125-147 (2014).

42. R. P. Haberman, A. Branch, M. Gallagher, Targeting Neural Hyperactivity as a Treatment to Stem Progression of Late-Onset Alzheimer's Disease., Neurotherapeutics 14, 662-676 (2017).

43. M. A. Yassa, S. M. Stark, A. Bakker, M. S. Albert, M. Gallagher, C. E. L. Stark, High-resolution structural and functional MRI of hippocampal CA3 and dentate gyrus in patients with amnestic Mild Cognitive Impairment, Neuroimage 51, 1242-1252 (2010).

44. R. Fontana, M. Agostini, E. Murana, M. Mahmud, E. Scremin, M. Rubega, G. Sparacino, S. Vassanelli, C. Fasolato, Early hippocampal hyperexcitability in PS2APP mice: role of mutant PS2 and APP, Neurobiol. Aging 50, 64-76 (2017).

45. J. J. Palop, J. Chin, E. D. Roberson, J. Wang, M. T. Thwin, N. Bien-Ly, J. Yoo, K. O. Ho, G.-Q. Yu, A. Kreitzer, S. Finkbeiner, J. L. Noebels, L. Mucke, Aberrant excitatory neuronal activity and compensatory remodeling of inhibitory hippocampal circuits in mouse models of Alzheimer's disease., Neuron 55, 697711 (2007).

46. R. P. Brenner, R. F. Ulrich, D. G. Spiker, R. J. Sclabassi, C. F. Reynolds, R. S. Marin, F. Boller, Computerized EEG spectral analysis in elderly normal, demented and depressed subjects., Electroencephalogr. Clin. Neurophysiol. 64, 483-92 (1986).

47. C. E. Jackson, P. J. Snyder, Electroencephalography and event-related potentials as biomarkers of mild cognitive impairment and mild Alzheimer's disease, Alzheimer's Dement. 4, S137-S143 (2008).

48. J. Jeong, EEG dynamics in patients with Alzheimer's disease, Clin. Neurophysiol. 115, 1490-1505 (2004). 
49. D. Z. Milikovsky, I. Weissberg, L. Kamintsky, K. Lippmann, O. Schefenbauer, F. Frigerio, M. Rizzi, L. Sheintuch, D. Zelig, J. Ofer, A. Vezzani, A. Friedman, Electrocorticographic Dynamics as a Novel Biomarker in Five Models of Epileptogenesis, J. Neurosci. 37, 4450-4461 (2017).

50. C. Wachinger, D. H. Salat, M. Weiner, M. Reuter, Alzheimer's Disease Neuroimaging Initiative, Whole-brain analysis reveals increased neuroanatomical asymmetries in dementia for hippocampus and amygdala, Brain 139, 3253-3266 (2016).

51. L. deToledo-Morrell, T. R. Stoub, M. Bulgakova, R. S. Wilson, D. A. Bennett, S. Leurgans, J. Wuu, D. A. Turner, MRI-derived entorhinal volume is a good predictor of conversion from MCl to AD., Neurobiol. Aging 25, 1197-203 (2004).

52. Y. Wang, S. L. Risacher, J. D. West, B. C. McDonald, T. R. MaGee, M. R. Farlow, S. Gao, D. P. O’Neill, A. J. Saykin, Altered Default Mode Network Connectivity in Older Adults with Cognitive Complaints and Amnestic Mild Cognitive Impairment, J. Alzheimer's Dis. 35, 751-760 (2013).

53. C. Yang, S. Zhong, X. Zhou, L. Wei, L. Wang, S. Nie, The Abnormality of Topological Asymmetry between Hemispheric Brain White Matter Networks in Alzheimer's Disease and Mild Cognitive Impairment, Front. Aging Neurosci. 9, 261 (2017).

54. L. Yue, T. Wang, J. Wang, G. Li, J. Wang, X. Li, W. Li, M. Hu, S. Xiao, Asymmetry of Hippocampus and Amygdala Defect in Subjective Cognitive Decline Among the Community Dwelling Chinese, Front. Psychiatry 9, 226 (2018).

55. N. Cherbuin, C. Réglade-Meslin, R. Kumar, P. Sachdev, K. J. Anstey, Mild cognitive disorders are associated with different patterns of brain asymmetry than normal aging: the PATH through life study, Front. Psychiatry 1, 11 (2010).

56. J. Lonsdale, J. Thomas, M. Salvatore, R. Phillips, E. Lo, S. Shad, R. Hasz, G. Walters, F. Garcia, N. Young, B. Foster, M. Moser, E. Karasik, B. Gillard, K. Ramsey, S. Sullivan, J. Bridge, H. Magazine, J. Syron, J. Fleming, L. Siminoff, H. Traino, M. Mosavel, L. Barker, S. Jewell, D. Rohrer, D. Maxim, D. Filkins, P. Harbach, E. Cortadillo, B. Berghuis, L. Turner, E. Hudson, K. Feenstra, L. Sobin, J. Robb, P. Branton, G. Korzeniewski, C. Shive, D. Tabor, L. Qi, K. Groch, S. Nampally, S. Buia, A. Zimmerman, A. Smith, R. Burges, K. Robinson, K. Valentino, D. Bradbury, M. Cosentino, N. Diaz-Mayoral, M. Kennedy, T. Engel, P. Williams, K. Erickson, K. Ardlie, W. Winckler, G. Getz, D. DeLuca, D. MacArthur, M. Kellis, A. Thomson, T. Young, E. Gelfand, M. Donovan, Y. Meng, G. Grant, D. Mash, Y. Marcus, M. Basile, J. Liu, J. Zhu, Z. Tu, N. J. Cox, D. L. Nicolae, E. R. Gamazon, H. K. Im, A. Konkashbaev, J. Pritchard, M. Stevens, T. Flutre, X. Wen, E. T. Dermitzakis, T. Lappalainen, R. Guigo, J. Monlong, M. Sammeth, D. Koller, A. Battle, S. Mostafavi, M. McCarthy, M. Rivas, J. Maller, I. Rusyn, A. Nobel, F. Wright, A. Shabalin, M. Feolo, N. Sharopova, A. Sturcke, J. Paschal, J. M. Anderson, E. L. Wilder, L. K. Derr, E. D. Green, J. P. Struewing, G. Temple, S. Volpi, J. T. Boyer, E. J. Thomson, M. S. Guyer, C. Ng, A. Abdallah, D. Colantuoni, T. R. Insel, S. E. Koester, A. R. Little, P. K. Bender, T. Lehner, Y. Yao, C. C. Compton, J. B. Vaught, S. Sawyer, N. C. Lockhart, J. Demchok, H. F. Moore, The Genotype-Tissue Expression (GTEx) project, Nat. Genet. 45, 580-585 (2013).

57. R. M. J. Deacon, J. N. P. Rawlins, T-maze alternation in the rodent., Nat. Protoc. 1, 7-12 (2006).

58. R. Lalonde, The neurobiological basis of spontaneous alternation., Neurosci. Biobehav. Rev. 26, 91104 (2002).

59. D. M. Bannerman, J. N. P. Rawlins, S. B. McHugh, R. M. J. Deacon, B. K. Yee, T. Bast, W.-N. Zhang, H. H. J. Pothuizen, J. Feldon, Regional dissociations within the hippocampus--memory and anxiety., Neurosci. Biobehav. Rev. 28, 273-83 (2004). 
60. D. Reisel, D. M. Bannerman, W. B. Schmitt, R. M. J. Deacon, J. Flint, T. Borchardt, P. H. Seeburg, J. N. P. Rawlins, Spatial memory dissociations in mice lacking GluR1., Nat. Neurosci. 5, 868-73 (2002).

61. C. Rabender, E. Mezzaroma, A. G. Mauro, R. Mullangi, A. Abbate, M. Anscher, B. Hart, R. Mikkelsen, IPW-5371 Proves Effective as a Radiation Countermeasure by Mitigating Radiation-Induced Late Effects, Radiat. Res. 186, 478-488 (2016).

62. S. N. Burke, J. L. Wallace, S. Nematollahi, A. R. Uprety, C. A. Barnes, Pattern separation deficits may contribute to age-associated recognition impairments., Behav. Neurosci. 124, 559-573 (2010).

63. T. Murai, S. Okuda, T. Tanaka, H. Ohta, Characteristics of object location memory in mice: Behavioral and pharmacological studies., Physiol. Behav. 90, 116-24 (2007).

64. N. A. Bishop, T. Lu, B. A. Yankner, Neural mechanisms of ageing and cognitive decline, Nature 464, 529-535 (2010).

65. J. H. Morrison, M. G. Baxter, The ageing cortical synapse: hallmarks and implications for cognitive decline., Nat. Rev. Neurosci. 13, 240-50 (2012).

66. L. Erraji-Benchekroun, M. D. Underwood, V. Arango, H. Galfalvy, P. Pavlidis, P. Smyrniotopoulos, J. J. Mann, E. Sibille, Molecular aging in human prefrontal cortex is selective and continuous throughout adult life, Biol. Psychiatry 57, 549-558 (2005).

67. T. Lu, Y. Pan, S.-Y. Y. Kao, C. Li, I. Kohane, J. Chan, B. A. Yankner, Gene regulation and DNA damage in the ageing human brain, Nature 429, 883-891 (2004).

68. R. P. Haberman, C. Colantuoni, A. M. Stocker, A. C. Schmidt, J. T. Pedersen, M. Gallagher, Prominent hippocampal CA3 gene expression profile in neurocognitive aging, Neurobiol. Aging 32, 1678-1692 (2011).

69. A. D. Lam, G. Deck, A. Goldman, E. N. Eskandar, J. Noebels, A. J. Cole, Silent hippocampal seizures and spikes identified by foramen ovale electrodes in Alzheimer's disease., Nat. Med. 23, 678-680 (2017).

70. K. A. Vossel, K. G. Ranasinghe, A. J. Beagle, D. Mizuiri, S. M. Honma, A. F. Dowling, S. M. Darwish, V. Van Berlo, D. E. Barnes, M. Mantle, A. M. Karydas, G. Coppola, E. D. Roberson, B. L. Miller, P. A. Garcia, H. E. Kirsch, L. Mucke, S. S. Nagarajan, Incidence and impact of subclinical epileptiform activity in Alzheimer's disease, Ann. Neurol. (2016), doi:10.1002/ana.24794.

71. K. A. Vossel, A. J. Beagle, G. D. Rabinovici, H. Shu, S. E. Lee, G. Naasan, M. Hegde, S. B. Cornes, M. L. Henry, A. B. Nelson, W. W. Seeley, M. D. Geschwind, M. L. Gorno-Tempini, T. Shih, H. E. Kirsch, P. A. Garcia, B. L. Miller, L. Mucke, Seizures and epileptiform activity in the early stages of Alzheimer disease., JAMA Neurol. 70, 1158-66 (2013).

72. J. J. Palop, L. Mucke, Epilepsy and Cognitive Impairments in Alzheimer Disease, Arch. Neurol. 66, 435 (2009).

73. J. Chin, H. E. Scharfman, Shared cognitive and behavioral impairments in epilepsy and Alzheimer's disease and potential underlying mechanisms, Epilepsy Behav. 26, 343-351 (2013).

74. K. Kam, Á. M. Duffy, J. Moretto, J. J. LaFrancois, H. E. Scharfman, Interictal spikes during sleep are an early defect in the Tg2576 mouse model of $\beta$-amyloid neuropathology., Sci. Rep. 6, 20119 (2016).

75. J. Cloyd, W. Hauser, A. Towne, R. Ramsay, R. Mattson, F. Gilliam, T. Walczak, Epidemiological and medical aspects of epilepsy in the elderly, Epilepsy Res. 68, 39-48 (2006). 
76. W. A. Hauser, J. F. Annegers, L. T. Kurland, Incidence of epilepsy and unprovoked seizures in Rochester, Minnesota: 1935-1984., Epilepsia 34, 453-68.

77. S. C. Schachter, G. W. Cramer, G. D. Thompson, R. J. Chaponis, M. A. Mendelson, L. Lawhorne, An evaluation of antiepileptic drug therapy in nursing facilities., J. Am. Geriatr. Soc. 46, 1137-41 (1998).

78. M. P. Mattson, T. Magnus, Ageing and neuronal vulnerability, Nat. Rev. Neurosci. 7, 278-294 (2006).

79. Q. Shi, K. J. Colodner, S. B. Matousek, K. Merry, S. Hong, J. E. Kenison, J. L. Frost, K. X. Le, S. Li, J.-C. Dodart, B. J. Caldarone, B. Stevens, C. A. Lemere, Complement C3-Deficient Mice Fail to Display AgeRelated Hippocampal Decline, J. Neurosci. 35, 13029-13042 (2015).

80. M. Yeoman, G. Scutt, R. Faragher, Insights into CNS ageing from animal models of senescence, Nat. Rev. Neurosci. 13, 435-445 (2012).

81. H. Chow, K. Herrup, Genomic integrity and the ageing brain, Nat. Rev. Neurosci. 16, 672-684 (2015).

82. K. Herrup, The case for rejecting the amyloid cascade hypothesis., Nat. Neurosci. 18, 794-9 (2015).

83. B. Cameron, G. E. Landreth, Inflammation, microglia, and alzheimer's disease, Neurobiol. Dis. 37, 503-509 (2010).

84. W.-S. Chung, C. A. Welsh, B. A. Barres, B. Stevens, Do glia drive synaptic and cognitive impairment in disease?, Nat. Neurosci. 18, 1539-1545 (2015).

85. S. A. Liddelow, K. A. Guttenplan, L. E. Clarke, F. C. Bennett, C. J. Bohlen, L. Schirmer, M. L. Bennett, A. E. Münch, W.-S. Chung, T. C. Peterson, D. K. Wilton, A. Frouin, B. A. Napier, N. Panicker, M. Kumar, M. S. Buckwalter, D. H. Rowitch, V. L. Dawson, T. M. Dawson, B. Stevens, B. A. Barres, Neurotoxic reactive astrocytes are induced by activated microglia, Nature 541, 481-487 (2017).

86. L. Soreq, J. UK Brain Expression Consortium, E. North American Brain Expression Consortium, J. Rose, E. Soreq, J. Hardy, D. Trabzuni, M. R. Cookson, C. Smith, M. Ryten, R. Patani, J. Ule, Major Shifts in Glial Regional Identity Are a Transcriptional Hallmark of Human Brain Aging., Cell Rep. 18, 557-570 (2017).

87. L. E. Clarke, S. A. Liddelow, C. Chakraborty, A. E. Münch, M. Heiman, B. A. Barres, Normal aging induces A1-like astrocyte reactivity., Proc. Natl. Acad. Sci. U. S. A. 115, E1896-E1905 (2018).

88. S. J. Chinta, G. Woods, M. Demaria, A. Rane, Y. Zou, A. McQuade, S. Rajagopalan, C. Limbad, D. T. Madden, J. Campisi, J. K. Andersen, Cellular Senescence Is Induced by the Environmental Neurotoxin Paraquat and Contributes to Neuropathology Linked to Parkinson's Disease, Cell Rep. 22, 930-940 (2018).

89. J. Yan, H. Zhang, Y. Yin, J. Li, Y. Tang, S. Purkayastha, L. Li, D. Cai, Obesity- and aging-induced excess of central transforming growth factor- $\beta$ potentiates diabetic development via an RNA stress response., Nat. Med. 20, 1001-8 (2014).

90. J. R. Pineda, M. Daynac, A. Chicheportiche, A. Cebrian-Silla, K. Sii Felice, J. M. Garcia-Verdugo, F. D. Boussin, M.-A. Mouthon, Vascular-derived TGF- $\beta$ increases in the stem cell niche and perturbs neurogenesis during aging and following irradiation in the adult mouse brain, EMBO Mol. Med. 5, 548562 (2013).

91. Y. H. El-Hayek, C. Wu, H. Ye, J. Wang, P. L. Carlen, L. Zhang, Hippocampal excitability is increased in aged mice, Exp. Neurol. 247, 710-719 (2013). 
92. K. P. Doyle, E. Cekanaviciute, L. E. Mamer, M. S. Buckwalter, TGF $\beta$ signaling in the brain increases with aging and signals to astrocytes and innate immune cells in the weeks after stroke, $J$. Neuroinflammation 7, 62 (2010).

93. J. E. Tichauer, B. Flores, B. Soler, L. Eugenín-von Bernhardi, G. Ramírez, R. von Bernhardi, Agedependent changes on TGF $\beta 1$ Smad3 pathway modify the pattern of microglial cell activation, Brain. Behav. Immun. 37, 187-196 (2014).

94. H. Yousef, M. J. Conboy, A. Morgenthaler, C. Schlesinger, L. Bugaj, P. Paliwal, C. Greer, I. M. Conboy, D. Schaffer, Systemic attenuation of the TGF- $\beta$ pathway by a single drug simultaneously rejuvenates hippocampal neurogenesis and myogenesis in the same old mammal, Oncotarget 6, 11959-11978 (2015).

95. Y. Chassidim, R. Veksler, S. Lublinsky, G. S. Pell, A. Friedman, I. Shelef, Quantitative imaging assessment of blood-brain barrier permeability in humans., Fluids Barriers CNS 10, 9 (2013).

96. R. Veksler, I. Shelef, A. Friedman, Blood-Brain Barrier Imaging in Human Neuropathologies., Arch. Med. Res. (2014), doi:10.1016/j.arcmed.2014.11.016.

97. I. Weissberg, R. Veksler, L. Kamintsky, R. Saar-Ashkenazy, D. Z. Milikovsky, I. Shelef, A. Friedman, Imaging blood-brain barrier dysfunction in football players., JAMA Neurol. 71, 1453-5 (2014).

98. I. Weissberg, A. Reichert, U. Heinemann, A. Friedman, Blood-brain barrier dysfunction in epileptogenesis of the temporal lobe., Epilepsy Res. Treat. 2011, 143908 (2011).

99. E. Seiffert, J. P. Dreier, S. Ivens, I. Bechmann, O. Tomkins, U. Heinemann, A. Friedman, Lasting bloodbrain barrier disruption induces epileptic focus in the rat somatosensory cortex., J. Neurosci. 24, 782936 (2004).

100. S. Sharma, S. Rakoczy, H. Brown-Borg, Assessment of spatial memory in mice, Life Sci. 87, 521-536 (2010).

\section{Acknowledgements}

This research was supported by NSF GRFP fellowships (V.V.S. and A.R.F.); Siebel Fellowship (V.V.S); NIH NRSA fellowship F31AG054147 (A.R.F.); NIH grants R01NS066005 and R56NS066005, a Bakar Foundation Fellowship, the Archer Foundation Award (D.K.); the European Union's Seventh Framework Program (FP7/2007-2013, grant agreement 602102, EPITARGET), the Israel Science Foundation (717/15) (A.F.); $\mathrm{NIH}$ grant R01AG042679 (A.D.); and the Binational Israel-USA Science Foundation (D.K. and A.F.). We thank Kelley Patten, Ami Citri, Hermona Soreq, and Inbal Goshen for edits on the manuscript. We thank Su Lee for graphic design of the schematic diagram. 
Figures and Legends

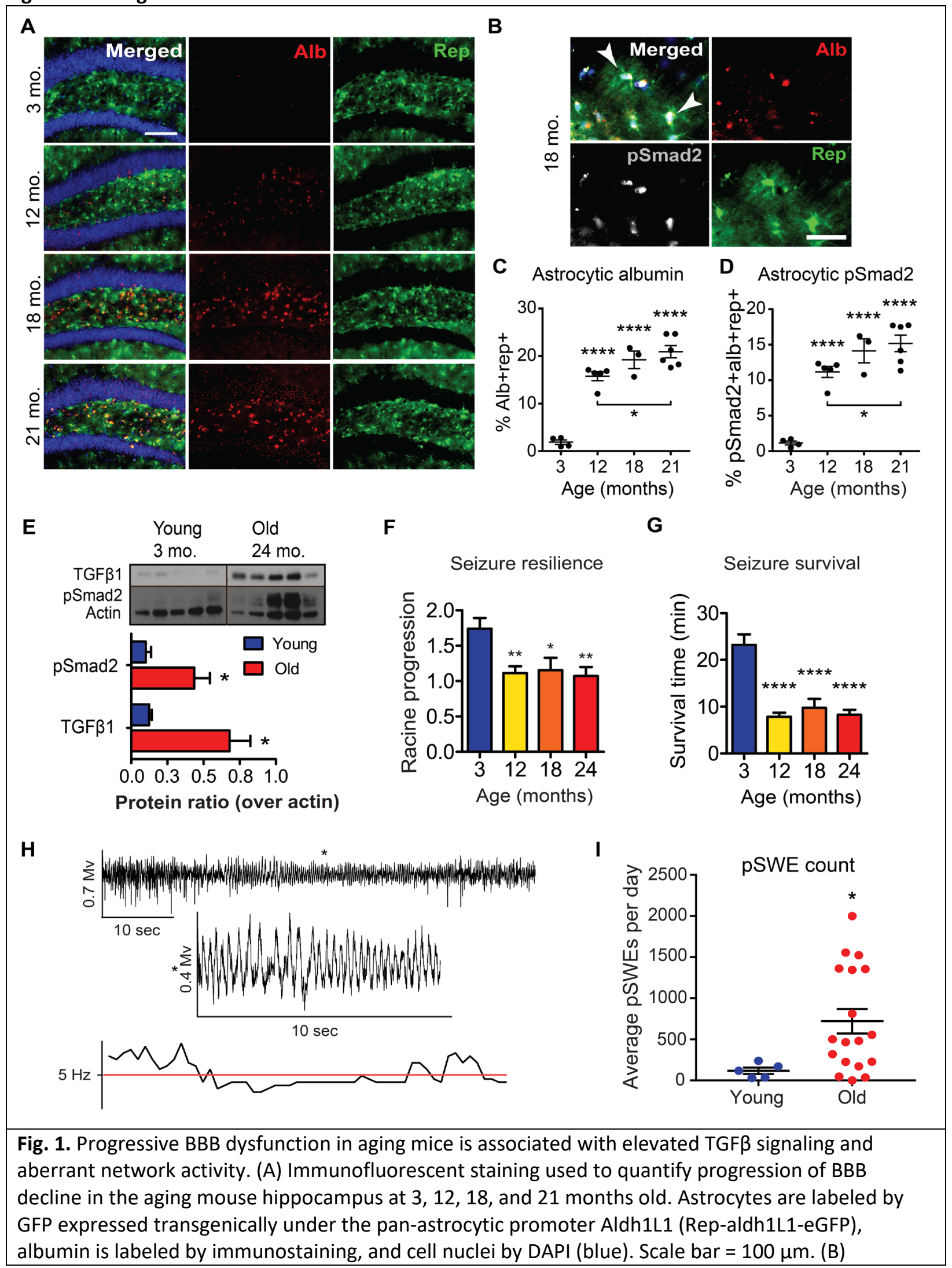


Immunostaining was used to investigate co-localization of albumin and pSmad2 (arrows) in Repaldh1L1-eGFP astrocytes (Scale bar $=30 \mu \mathrm{m}$ ). (C) Albumin localized in astrocytes increases with age (ANOVA, $p<0.0001$ ). (D) Aging mice show an increase in the level of $p S m a d 2$ colabeled with albumin in astrocytes (ANOVA, $p=<0.0001$ ). For all measures, groups were compared by Bonferroni post-hoc test. Sample sizes are $n=4$ (3 mo); 5 (12 mo); 3 (18 mo); and 6 (21 mo). (E) Western blot analyzing TGF $\beta 1$ and pSmad2, outputs of the TGF $\beta$ signaling pathway, in hippocampus from young and old mice. Western blot densitometry shows that pSmad2 (t-test, $p=0.019$ ) and TGF $\beta$ (t-test with Welch's correction, $p=0.018$ ) protein levels were elevated in the aged mouse hippocampus $(n=5)$. (F) At 3 , 12,18 , and 24 months seizures were induced in mice by PTZ injection to assay hyperexcitability. Seizure severity in mice was quantified by fitting linear regression slopes to measures of progression through each stage of seizure in the modified Racine scale. Aged mice (12-24 months) had decreased linear regression slopes indicating faster progression through all stages of seizure severity (1-way ANOVA, $p=0.0011$ with Bonferroni posttest; $n=13$ ( $3 \mathrm{mo}$ ), 10 (12 mo), 8 (18 mo and $24 \mathrm{mo})$ ). (G) Latency to mortality caused by severe seizures was significantly faster in 12-24 month old groups compared to young mice (1-way ANOVA, $p<0.0001$ with Bonferroni posttest). (H) A representative trace shows a pSWE with slow-wave activity less than $5 \mathrm{~Hz}$ within a 10 second window (marked with *). (I) Aged mice showed a significantly elevated number of pSWEs compared to young mice. For all tests, $* p<0.05, * * p<0.01, * * * p<0.005, * * * * p<0.001$. 
A

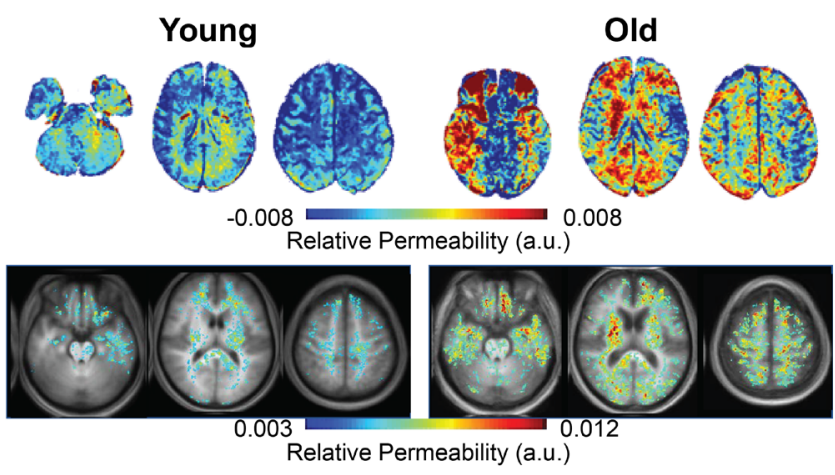

B Permeability in right hippocampus

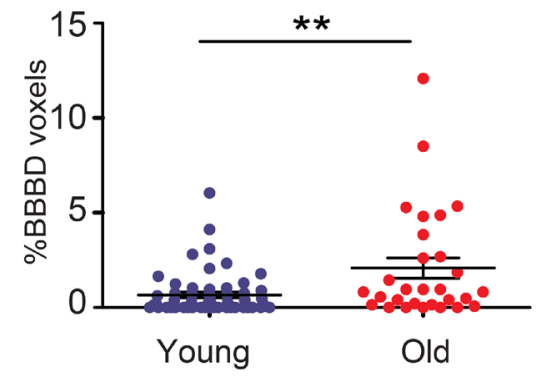

D
C

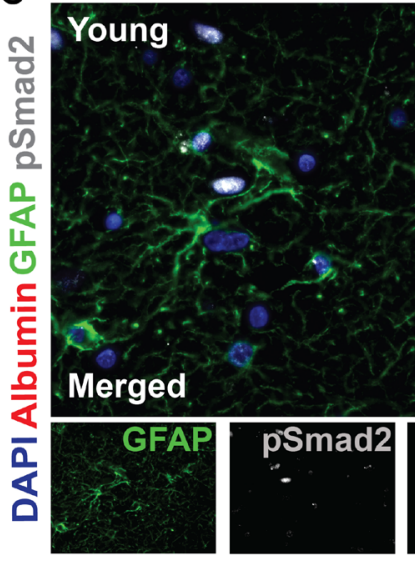

$\mathbf{E}$

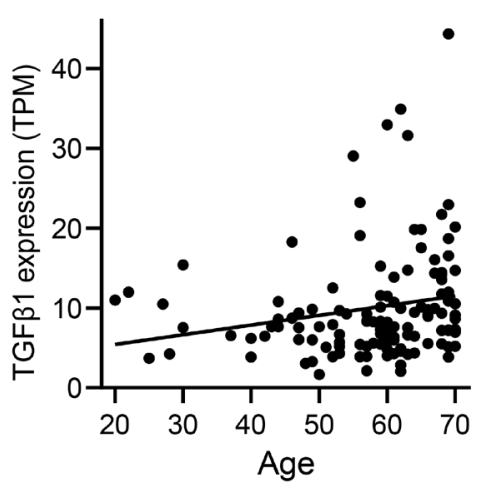

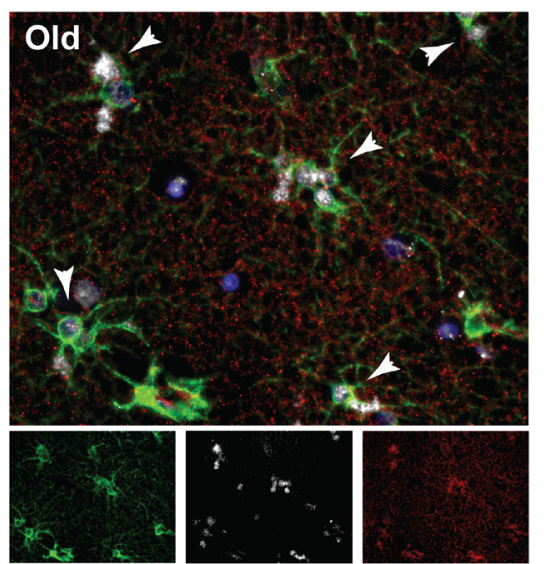

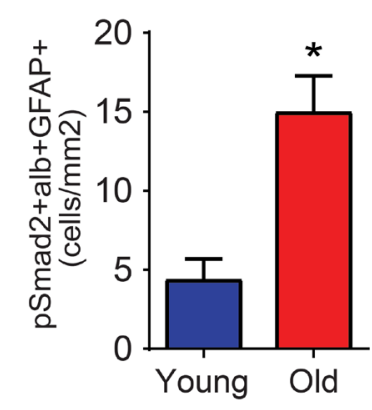

Fig. 2. Aging patients show decline in the integrity of the BBB and increased TGF $\beta$ signaling. (A) (Top Panel): Representative images from DCE-MRI scans of a young (30 years old) and old ( 70 years old) subject using an MRI-sensitive contrast agent, Gd-DTPA, which does not cross the intact BBB. Intensity of signal reflects relative BBB permeability (in arbitrary units, a.u.). (Bottom Panel): Average permeability maps from all young (ages 20-40) and old (60-80) subjects. (B) BBB permeability was significantly elevated in the right hippocampus of old subjects compared to young. (C) Representative images of immunofluorescent staining of post-mortem human hippocampal tissue from young (31.3 \pm 5 years) and old ( $70.6 \pm 5.6$ years) individuals, showing albumin in the aging hippocampus, localized in astrocytes (GFAP) and co-localized with pSmad2, indicated by arrows. Nuclei are visualized by DAPI stain. Scale bar $=50 \mu \mathrm{m}$. (D) The aging human hippocampus has significantly elevated numbers of 
astrocytes that colabel with albumin and pSmad2 (t-test, $p=0.0324, n=3$ young, 10 old). (E) Scatterplots and best-fit linear regressions of TGF $\beta$ expression levels (transcripts per million; TPM) from hippocampus of human subjects aged $20-70$ years old ( $n=123$ subjects), accessed from the GTEx project expression database. Aging is significantly correlated with increasing hippocampal expression of TGF $\beta 1$ (Pearson correlation, $r=0.195, p=0.031)$ and TGF $\beta 3(r=0.184,=0.042)$ isoforms, but not TGF $\beta 2(r=-0.028)$.

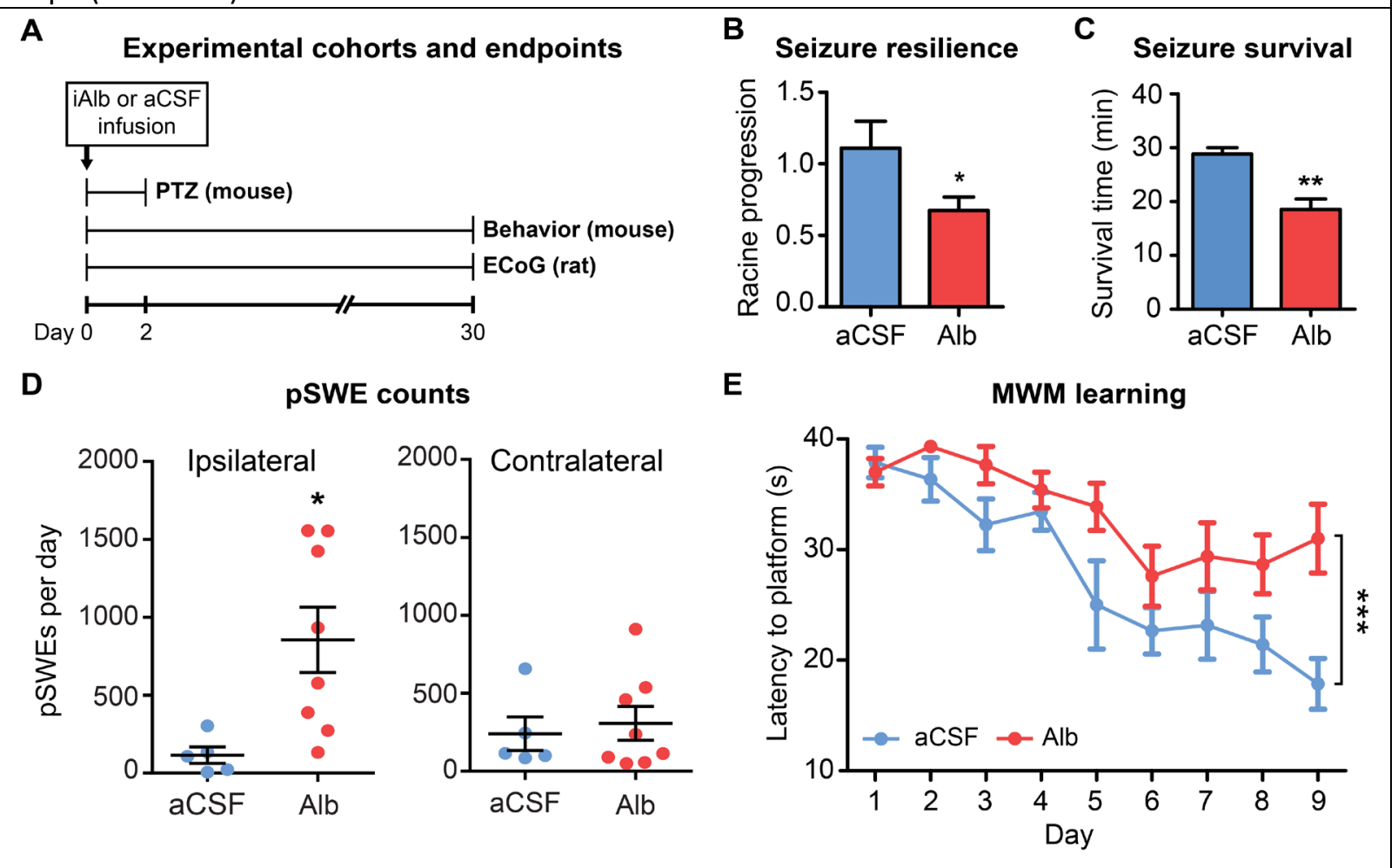

Fig. 3. Induction of TGF $\beta$ signaling in young rodents causes aberrant network activity, vulnerability to induced seizures, and cognitive impairment. (A) Timeline of outcome measures (endpoints) taken in cohorts of young adult rats and mice after icv albumin infusion (iAlb). (B) Young mice received iAlb or aCSF control infusion for 48 hours, followed by PTZ seizure induction. Albumin infusion caused increased severity in induced seizures with regression slopes showing faster seizure progression ( $t$ test with Welch's correction, $p=0.0497, n=4)$, and $(C)$ faster latency to mortality (t-test, $p=0.004$ ), compared to aCSF controls. (D) ECoG activity was recorded from rats following one month of iAlb infusion. iAlb rats had significantly elevated numbers of pSWEs compared to aCSF-infused controls in the ipsilateral hemisphere receiving infusion, but not the contralateral hemisphere. (E) Young mice were given iAlb surgery and then tested in the MWM task one month later. Compared to aCSF controls, iAlb mice had significantly poorer learning performance (repeated measure ANOVA, $n=9$ aCSF, $10 \mathrm{iAlb}$, main effect of learning over time $p<0.0001$, main effect of group $p=0.0093$, with Bonferroni posttest on day 9 showing significant differences between aCSF and iAlb, $p<0.001$ ). For all tests, ${ }^{*} \mathrm{p}<0.05,{ }^{* *} \mathrm{p}<0.01,{ }^{* * *} \mathrm{p}<0.005, * * * * \mathrm{p}<0.001$. 
A

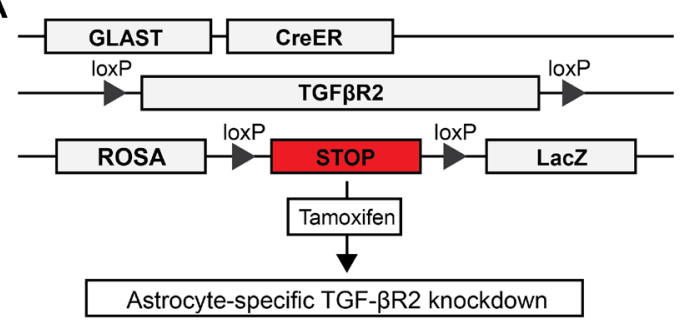

C
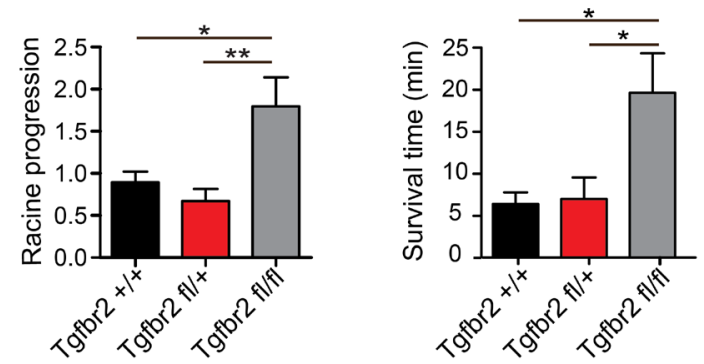

B

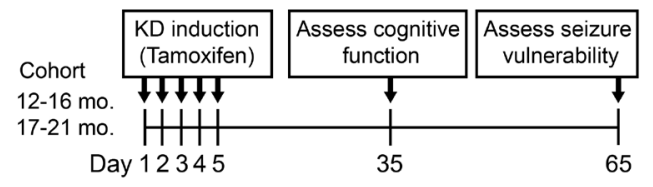

D Seizure resilience during late aging
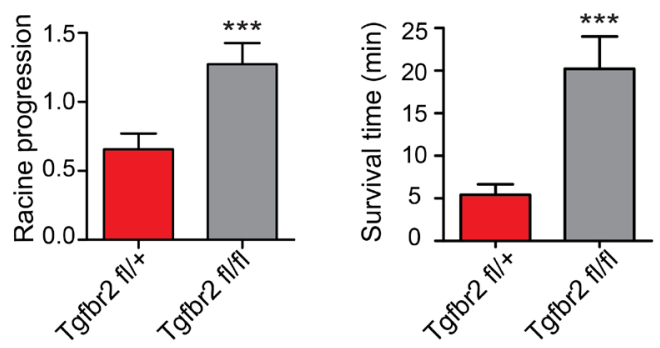

E

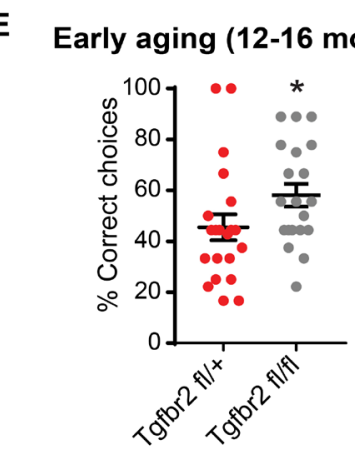

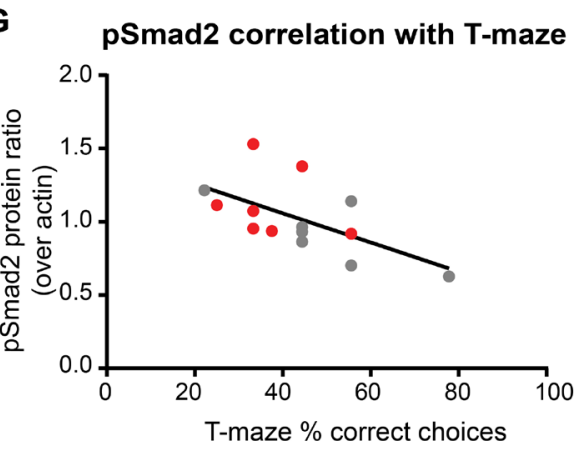

Fig. 4. Knockdown of astrocytic TGF $\beta$ reverses neurological outcomes in aging mice. (A) Schematic of the transgenic aTGF $\beta R$ KD system. The astrocytic GLAST promoter drives expression of Cre recombinase in astrocytes. Following induction with tamoxifen injection, activated Cre excises the TGF $\beta$ R gene at inserted floxed ( $\mathrm{fl}$ ) loxP sites. LacZ reporter expression provides a readout of Cre activity. (B) Experimental timeline. KD was induced in early (12-16 mo.) and late (17-24 mo.) aged mice, and T-maze testing was performed 35 days later. At 65 days post-induction, mice were tested for vulnerability to PTZ-induced seizures. (C) aTGF $\beta R$ KD $\left(\right.$ Tgfbr $\left.{ }^{\mathrm{fl} / \mathrm{fl}}\right)$ reversed seizure vulnerability in 12-16 mo old mice, significantly slowing progression through the Racine scale (1-way ANOVA, $p=$ 0.0019 , with Bonferroni posttest, $\mathrm{n}=5 \mathrm{Tgfbr}^{+/+}, 6 \mathrm{Tgfbr}^{\mathrm{fl} /+}$ and $\mathrm{Tgfbr} 2^{\mathrm{fl} / \mathrm{fl}}$ ) and latency to mortality (1way ANOVA, $p=0.022$, with Bonferroni posttest), compared to control heterozygous mice (Tgfbr $2^{\mathrm{fl} /+}$ ) and mice given oil control rather than tamoxifen induction $\left(\mathrm{Tgfbr}^{+/+}\right)$. (D) 17-24 month old mice with aTGF $\beta$ KD were similarly protected against PTZ seizure vulnerability with less severe seizures (t-test with Welch's correction, $\mathrm{p}=0.002, \mathrm{n}=5 \mathrm{Tgfbr} \mathrm{f}^{\mathrm{fl} / \mathrm{+}}$ and $9 \mathrm{Tgfbr}^{\mathrm{fl} / \mathrm{fl})}$ and delayed mortality (t-test with Welch's correction, $p=0.004)$. (E-F) In T-maze, aTGF $\beta R$ KD mice showed significantly better working memory performance compared to heterozygous controls at in both early aging (12-16 months old, Mann-Whitney test, $\mathrm{p}=0.0273, \mathrm{n}=21 \mathrm{Tgfbr}^{\mathrm{ff} /+}, 20 \mathrm{Tgfbr}^{\mathrm{fl} / \mathrm{fl}}$ ) and late aging assessments (t-test, $\mathrm{p}=$ $\left.0.035 \mathrm{n}=12 \mathrm{Tgfbr}^{\mathrm{fl} /+}, 11 \mathrm{Tgfbr}^{\mathrm{fl} / \mathrm{fl}}\right)$. (G) An additional cohort of 12-16 month old mice was tested in Tmaze, and hippocampi were dissected for Western blot analysis of TGF $\beta$ signaling (pSmad2) to assess 
individual outcomes. T-maze performance was negatively correlated with levels of pSmad2 signaling across individuals of both genotypes (Tgfbr2 $2^{\mathrm{fl} /+}$ and Tgfbr ${ }^{\mathrm{fl} / \mathrm{fl}}$ ) (Pearson's correlation, $r=-0.598, p=$ $0.024, \mathrm{n}=14)$. For all tests, $* \mathrm{p}<0.05, * * \mathrm{p}<0.01, * * * \mathrm{p}<0.005, * * * * \mathrm{p}<0.001$.

A

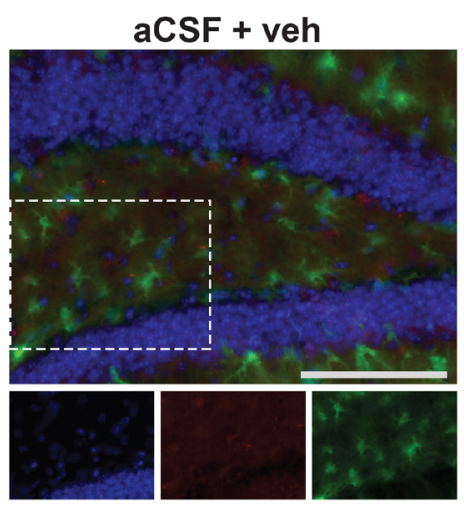

B

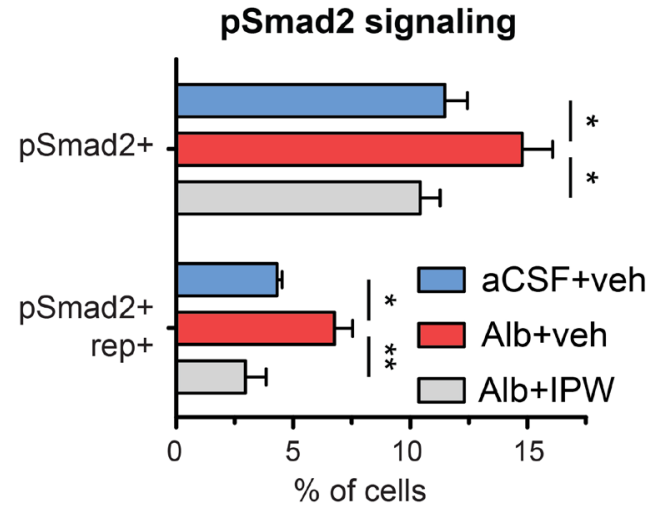

Alb + veh

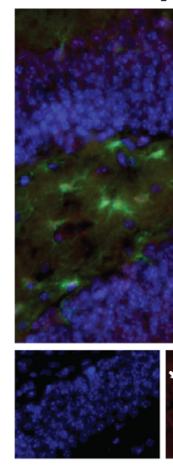

C

Seizure resilience

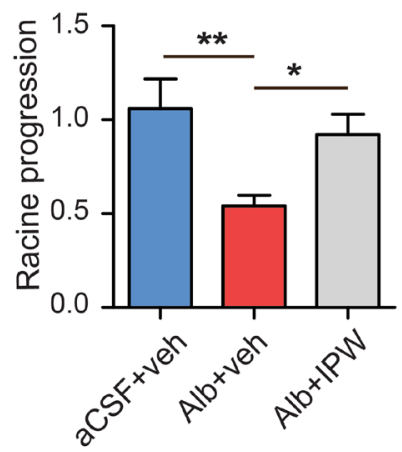

Alb + IPW

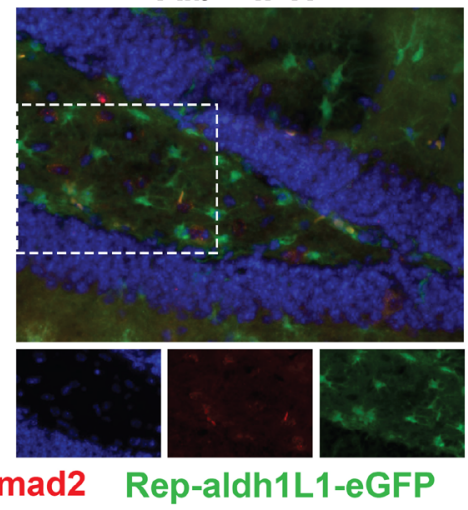

D

Seizure survival

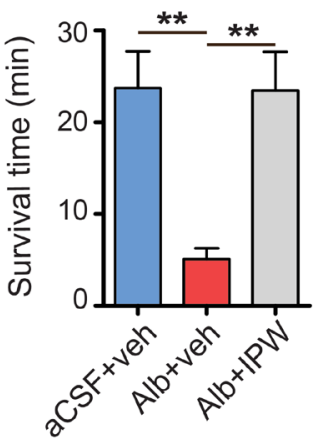

Fig. 5. IPW reduces TGF $\beta$ signaling and seizure vulnerability in young mice infused with albumin. (A) Immunofluorescent images from iAlb mice after treatment with IPW or vehicle injections during 7 days of icv albumin infusion (Scale bar $=100 \mu \mathrm{m}$; dotted box indicates region of inset image; arrows indicate examples of astrocytes colabeled with pSmad2). (B) iAlb mice showed elevated levels pSmad2 signaling localized in astrocytes (compared to aCSF controls) and IPW reduced pSmad2 (1way ANOVA with Neuman-Keuls Multiple Comparison Test: $\%$ pSmad2, $p=0.027 ; \%$ pSmad2+ rep,$+ p$ $=0.0048 ; n=6)$. (C) IPW treatment reduced seizure vulnerability conferred by iAlb, restoring seizure profile to aCSF control levels (1-way ANOVA, main effect of treatment $p=0.004$, with Bonferroni's posttest, $n=6$ (aCSF+veh and Alb+veh), 7 (Alb+IPW)). (D) IPW treatment also reversed the vulnerability to seizure mortality conferred by iAlb (1-way ANOVA with Tukey's posttest, $p=0.003, n=$ 6 (aCSF+veh and Alb+veh), 7 (Alb+IPW). For all tests, ${ }^{*} p<0.05,{ }^{* *} p<0.01,{ }^{* * *} p<0.005,{ }^{* * * *} p<0.001$. 


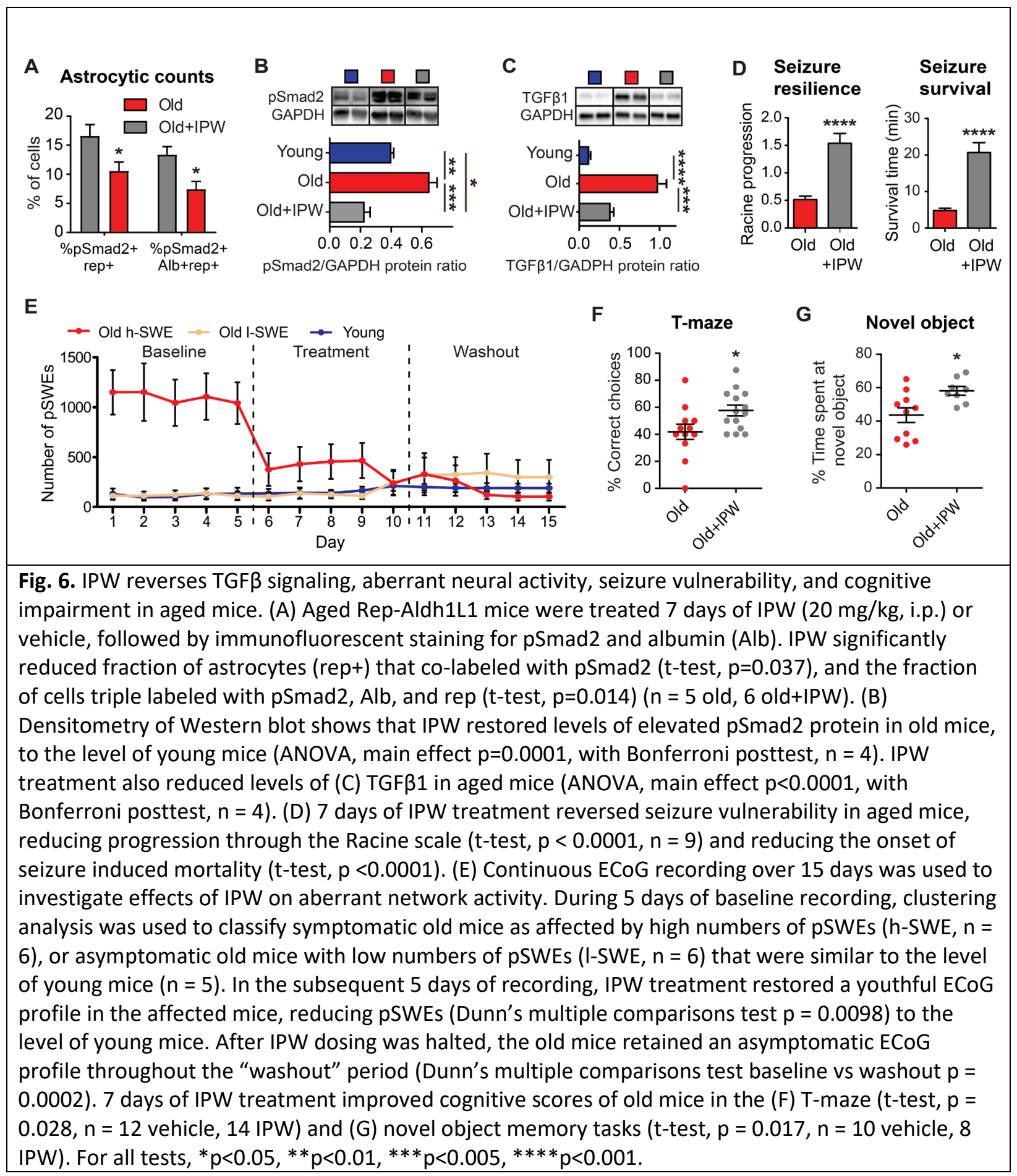




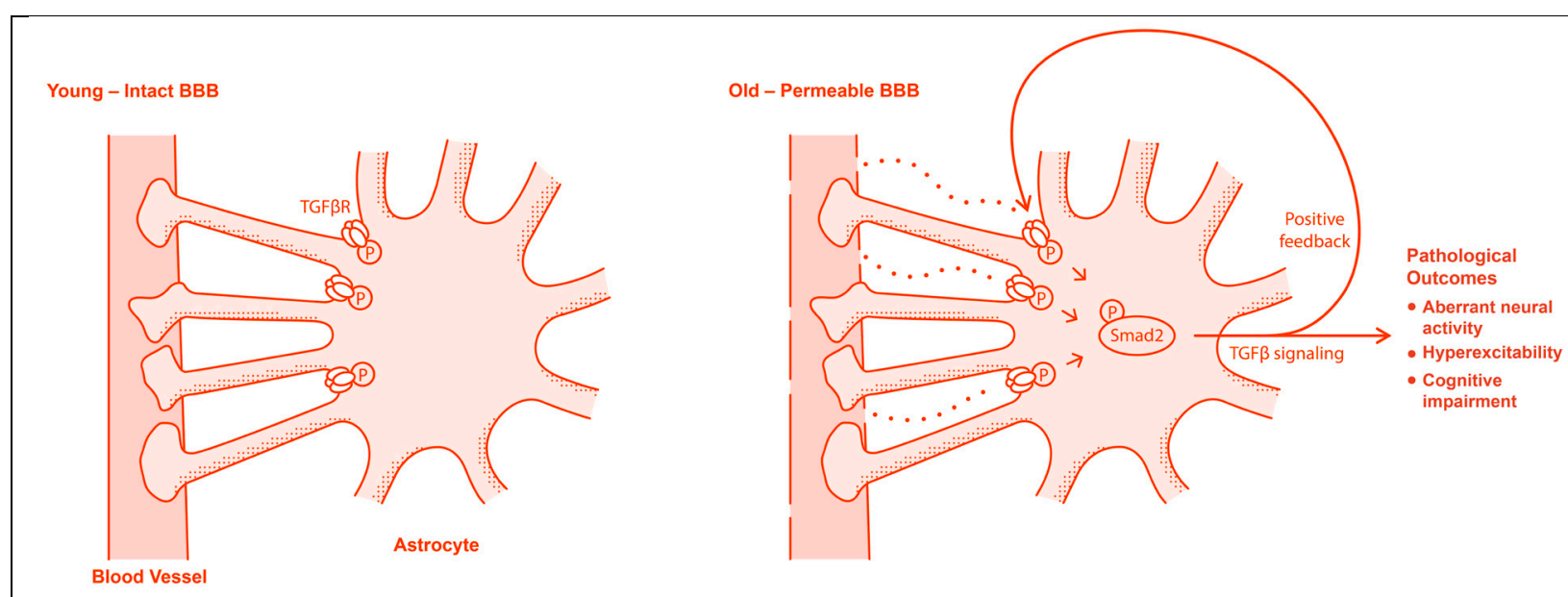

Fig. 7. Schematic summarizing the mechanistic model underlying BBB-related neural pathology. In old age, serum proteins including albumin cross the dysfunctional BBB and activate astrocytic TGF $\beta$ receptor (TGF $\beta$ ) signaling. Outputs of the astrocytic TGF $\beta$ signaling cascade include positive feedback and induction of symptomatic pathology including aberrant neural activity, hyperexcitability, and cognitive impairment. 